MATHEMATICS OF COMPUTATION

Volume 76, Number 259, July 2007, Pages 1425-1448

S 0025-5718(07)01946-1

Article electronically published on February 19, 2007

\title{
IDENTIFICATION OF SMALL INHOMOGENEITIES: ASYMPTOTIC FACTORIZATION
}

\author{
HABIB AMMARI, ROLAND GRIESMAIER, AND MARTIN HANKE
}

\begin{abstract}
We consider the boundary value problem of calculating the electrostatic potential for a homogeneous conductor containing finitely many small insulating inclusions. We give a new proof of the asymptotic expansion of the electrostatic potential in terms of the background potential, the location of the inhomogeneities and their geometry, as the size of the inhomogeneities tends to zero. Such asymptotic expansions have already been used to design direct (i.e. noniterative) reconstruction algorithms for the determination of the location of the small inclusions from electrostatic measurements on the boundary, e.g. MUSIC-type methods. Our derivation of the asymptotic formulas is based on integral equation methods. It demonstrates the strong relation between factorization methods and MUSIC-type methods for the solution of this inverse problem.
\end{abstract}

\section{INTRODUCTION}

Inverse boundary value problems for partial differential equations, in principle, are difficult to solve since they are both nonlinear and ill-posed. Recently new solution methods such as linear sampling methods and factorization methods have been developed which avoid the issue of nonlinearity. Basically, these methods make use of some sort of symmetric or self-adjoint factorization

$$
M=L F L^{*}
$$

of some (measurement) operator $M$. Then the idea, introduced first by Colton and Kirsch 20] (sampling method) and by Kirsch 29] (factorization method) in the context of inverse obstacle scattering problems, is to characterize the support of an obstacle by the range of some operator related to $M$. These methods have since then been applied to a variety of different applications; cf., e.g., the papers [17 19, 26] (sampling method) and [25, 28, 31, 32, 34] (factorization method), and the many references therein.

In order to handle the ill-posedness it is generally advisable to incorporate all available a priori knowledge about the unknown parameter and to try to determine very specific features. Embarking on this strategy the purpose could be to determine the location and size of diametrically small inclusions inside a homogeneous background. This situation arises for example in mine-detection and nondestructive testing. For this special case reconstruction methods for inverse boundary

Received by the editor January 7, 2006 and, in revised form, May 15, 2006.

2000 Mathematics Subject Classification. Primary 35R30, 35C20.

Key words and phrases. Electrical impedance tomography, small conductivity inhomogeneities, asymptotic expansions.

(C)2007 American Mathematical Society Reverts to public domain 28 years from publication 
value problems, which make use of asymptotic expansions of the solutions of the corresponding direct problems, have been developed during the last years. Among these, MUSIC-type algorithms, introduced by Devaney [23], seem to be very stable and therefore particularly useful for noisy data.

In this paper we consider an inverse boundary value problem for the Laplace equation as discussed in $[5,9,12,15,24$ in the case of small insulating inclusions. We prove an asymptotic expansion of the corresponding measurement operator similar to the asymptotic formulas in 24 but in a more functional analytic setting. Our proof is based on a factorization of the measurement operator developed in [11] and on layer potential techniques. We expand the three operators occurring in the factorization separately and use these expansions to calculate the leading order term in the asymptotic formula for the measurement operator. This way of proving the asymptotic expansion points out the strong relation between MUSIC-type algorithms and linear sampling methods explicitely (cf. also [16, 30]). Moreover, this method should be applicable to other inverse boundary value problems, where a factorization of the measurement operator is already available; cf., e.g. [26].

For closely related works concerning asymptotic expansions and reconstruction algorithms for inverse boundary value problems with diametrically small inclusions based on such expansions cf., e.g., [1 4, 7-10, 13, 14,37, the monograph 6 and the many references therein.

The outline of this paper is as follows. In Section 2 we introduce our notation and review the factorization of our measurement operator, i.e. of the difference of two Neumann-to-Dirichlet operators. Here and in the following three sections we restrict our derivations to the case of a single inclusion. Preliminary results concerning surface potentials are investigated in Section 3. In order to establish the asymptotic expansion we require some technical estimates and identities; these are found in Section 4. Then, in Section 5 , we derive our main result on the asymptotic factorization in the case of a single inclusion in Theorem 5.9 and its corollary. The case of multiple inclusions is treated in Section 6. Finally, in Section 7 we comment on how the asymptotics might be used in numerical computations.

\section{Factorization of the Neumann-to-Dirichlet operator}

Let $\Omega \subset \mathbb{R}^{d}, d \geq 2$, denote a bounded domain with boundary $\partial \Omega$ of class $C^{1, \alpha}$, $0<\alpha<1$. Suppose $\Omega$ contains a small inclusion $D_{\varepsilon}:=z+\varepsilon B$, where $B$ is a bounded $C^{1, \alpha}$ domain containing the origin. Here, the point $z \in \Omega$ determines the location of the inclusion and $B$ describes its relative shape. The inhomogeneity size is specified by the parameter $\varepsilon>0$ which is assumed to be small. We suppose that the domain $D_{\varepsilon}$ is well separated from the boundary, i.e. $\operatorname{dist}(z, \partial \Omega) \geq c_{0}$ for some constant $c_{0}>0$ and $\varepsilon$ is sufficiently small. Let $\nu$ denote the unit outward normal to the boundaries $\partial \Omega, \partial B$ and $\partial D_{\varepsilon}$, relative to $\Omega, B$ and $D_{\varepsilon}$, respectively.

In this section several results are stated without proof; these can be found in [11] or references therein.

Given a conductivity distribution of the form

$$
\sigma_{\varepsilon}(x):= \begin{cases}0, & \text { for } x \in D_{\varepsilon}, \\ 1, & \text { for } x \in \Omega \backslash \overline{D_{\varepsilon}}\end{cases}
$$


and a prescribed boundary current

$$
f \in H_{\diamond}^{-1 / 2}(\partial \Omega):=\left\{\phi \in H^{-1 / 2}(\partial \Omega) \mid \int_{\partial \Omega} \phi \mathrm{d} \sigma=0\right\},
$$

let $u_{\varepsilon}$ denote the electrostatic potential in the presence of the inclusion $D_{\varepsilon}$, i.e. the unique solution

$$
u_{\varepsilon} \in H_{\diamond, \partial \Omega}^{1}\left(\Omega \backslash \overline{D_{\varepsilon}}\right):=\left\{u \in H^{1}\left(\Omega \backslash \overline{D_{\varepsilon}}\right) \mid \int_{\partial \Omega} u \mathrm{~d} \sigma=0\right\}
$$

to

$$
\begin{aligned}
& \Delta u_{\varepsilon}=0, \quad \text { in } \Omega \backslash \overline{D_{\varepsilon}}, \\
& \frac{\partial u_{\varepsilon}}{\partial \nu}=f, \quad \text { on } \partial \Omega \text {, } \\
& \frac{\partial u_{\varepsilon}}{\partial \nu}=0, \quad \text { on } \partial D_{\varepsilon} .
\end{aligned}
$$

The background potential $u_{0}$ is the electrostatic potential for the same input current $f$ but without inclusions. That is, $u_{0}$ denotes the unique solution

$$
u_{0} \in H_{\diamond}^{1}(\Omega):=\left\{u \in H^{1}(\Omega) \mid \int_{\partial \Omega} u \mathrm{~d} \sigma=0\right\}
$$

to

$$
\begin{aligned}
& \Delta u_{0}=0, \quad \text { in } \Omega, \\
& \frac{\partial u_{0}}{\partial \nu}=f, \quad \text { on } \partial \Omega \text {. }
\end{aligned}
$$

The relations between the applied boundary current $f$ and the boundary voltages $\left.u_{\varepsilon}\right|_{\partial \Omega}$ and $\left.u_{0}\right|_{\partial \Omega}$ define two linear mappings

$$
\Lambda_{\varepsilon}: H_{\diamond}^{-1 / 2}(\partial \Omega) \rightarrow H_{\diamond}^{1 / 2}(\partial \Omega),\left.\quad f \mapsto u_{\varepsilon}\right|_{\partial \Omega},
$$

and

$$
\Lambda_{0}: H_{\diamond}^{-1 / 2}(\partial \Omega) \rightarrow H_{\diamond}^{1 / 2}(\partial \Omega),\left.\quad f \mapsto u_{0}\right|_{\partial \Omega},
$$

called the Neumann-to-Dirichlet operators associated with the two boundary value problems (2.1) and (2.2), respectively. Here,

$$
H_{\diamond}^{1 / 2}(\partial \Omega):=\left\{\phi \in H^{1 / 2}(\partial \Omega) \mid \int_{\partial \Omega} \phi \mathrm{d} \sigma=0\right\} .
$$

These mappings are in fact isomorphisms between these spaces.

In the following we want to examine the difference of the Neumann-to-Dirichlet operators $\Lambda_{\varepsilon}-\Lambda_{0}$. Therefore we introduce two additional boundary value problems and a diffraction problem: First consider the boundary value problem

$$
\begin{aligned}
& \Delta v_{\varepsilon}=0, \quad \text { in } \Omega \backslash \overline{D_{\varepsilon}}, \\
& \frac{\partial v_{\varepsilon}}{\partial \nu}=0, \quad \text { on } \partial \Omega, \\
& \frac{\partial v_{\varepsilon}}{\partial \nu}=\phi, \quad \text { on } \partial D_{\varepsilon},
\end{aligned}
$$


which for $\phi \in H_{\diamond}^{-1 / 2}\left(\partial D_{\varepsilon}\right)$ has a unique solution $v_{\varepsilon} \in H_{\diamond, \partial \Omega}^{1}\left(\Omega \backslash \overline{D_{\varepsilon}}\right)$. Thus, we may define

$$
L_{\varepsilon}: H_{\diamond}^{-1 / 2}\left(\partial D_{\varepsilon}\right) \rightarrow H_{\diamond}^{1 / 2}(\partial \Omega),\left.\quad \phi \mapsto v_{\varepsilon}\right|_{\partial \Omega},
$$

which is a bounded linear operator that takes Neumann data on $\partial D_{\varepsilon}$ and maps them onto the associated Dirichlet values on $\partial \Omega$. Recalling (2.1) and (2.2) we see that $L_{\varepsilon}\left(-\left.\frac{\partial u_{0}}{\partial \nu}\right|_{\partial D_{\varepsilon}}\right)=L_{\varepsilon}\left(\left.\frac{\partial u_{\varepsilon}}{\partial \nu}\right|_{\partial D_{\varepsilon}}-\left.\frac{\partial u_{0}}{\partial \nu}\right|_{\partial D_{\varepsilon}}\right)=\left.\left(u_{\varepsilon}-u_{0}\right)\right|_{\partial \Omega}$.

A short computation reveals that the dual operator $L_{\varepsilon}^{*}$ of $L_{\varepsilon}$ is defined via the solution of the problem

$$
\begin{aligned}
& \Delta v_{\varepsilon}^{*}=0, \\
& \text { in } \Omega \backslash \overline{D_{\varepsilon}} \text {, } \\
& \frac{\partial v_{\varepsilon}^{*}}{\partial \nu}=-\psi \text {, } \\
& \text { on } \partial \Omega \text {, } \\
& \frac{\partial v_{\varepsilon}^{*}}{\partial \nu}=0, \\
& \text { on } \partial D_{\varepsilon} \text {, }
\end{aligned}
$$

which for $\psi \in H_{\diamond}^{-1 / 2}(\partial \Omega)$ has a unique solution

$$
v_{\varepsilon}^{*} \in H_{\diamond, \partial D_{\varepsilon}}^{1}\left(\Omega \backslash \overline{D_{\varepsilon}}\right):=\left\{u \in H^{1}\left(\Omega \backslash \overline{D_{\varepsilon}}\right) \mid \int_{\partial D_{\varepsilon}} u \mathrm{~d} \sigma=0\right\},
$$

through

$$
L_{\varepsilon}^{*}: H_{\diamond}^{-1 / 2}(\partial \Omega) \rightarrow H_{\diamond}^{1 / 2}\left(\partial D_{\varepsilon}\right),\left.\quad \psi \mapsto v_{\varepsilon}^{*}\right|_{\partial D_{\varepsilon}} .
$$

Note that, apart from the normalization condition, (2.5) coincides with the boundary value problem (2.1), and hence $L_{\varepsilon}^{*} f=-\left.u_{\varepsilon}\right|_{\partial D_{\varepsilon}}+\left(1 /\left|\partial D_{\varepsilon}\right|\right) \int_{\partial D_{\varepsilon}} u_{\varepsilon} \mathrm{d} \sigma$, where $\left|\partial D_{\varepsilon}\right|$ denotes the surface measure of $\partial D_{\varepsilon}$.

Next consider the following diffraction problem with inhomogeneous jump condition:

$$
\begin{aligned}
\Delta w_{\varepsilon} & =0, & & \text { in } \Omega \backslash \partial D_{\varepsilon}, \\
\frac{\partial w_{\varepsilon}}{\partial \nu} & =0, & & \text { on } \partial \Omega, \\
{\left[w_{\varepsilon}\right]_{\partial D_{\varepsilon}} } & =\chi, & & \\
{\left[\frac{\partial w_{\varepsilon}}{\partial \nu}\right]_{\partial D_{\varepsilon}} } & =0, & &
\end{aligned}
$$

which for $\chi \in H_{\diamond}^{1 / 2}\left(\partial D_{\varepsilon}\right)$ has a unique solution $w_{\varepsilon}$ with $\left.w_{\varepsilon}\right|_{\Omega \backslash \overline{D_{\varepsilon}}} \in H_{\diamond, \partial \Omega}^{1}\left(\Omega \backslash \overline{D_{\varepsilon}}\right)$ and $\left.w_{\varepsilon}\right|_{D_{\varepsilon}} \in H^{1}\left(D_{\varepsilon}\right)$. Here, $[\cdot]_{\partial D_{\varepsilon}}$ denotes the difference between the respective traces from outside and inside the inner boundary $\partial D_{\varepsilon}$. Because of (2.7d),

$$
F_{\varepsilon}: H_{\diamond}^{1 / 2}\left(\partial D_{\varepsilon}\right) \rightarrow H_{\diamond}^{-1 / 2}\left(\partial D_{\varepsilon}\right), \quad \chi \mapsto-\left.\frac{\partial w_{\varepsilon}}{\partial \nu}\right|_{\partial D_{\varepsilon}},
$$

is a well-defined bounded linear operator. Especially for

$$
\chi:=-\left.u_{\varepsilon}\right|_{\partial D_{\varepsilon}}+\left(1 /\left|\partial D_{\varepsilon}\right|\right) \int_{\partial D_{\varepsilon}} u_{\varepsilon} \mathrm{d} \sigma
$$

the function

$$
w_{\varepsilon}:= \begin{cases}-u_{\varepsilon}+u_{0}, & \text { in } \Omega \backslash \overline{D_{\varepsilon}}, \\ u_{0}-\left(1 /\left|\partial D_{\varepsilon}\right|\right) \int_{\partial D_{\varepsilon}} u_{\varepsilon} \mathrm{d} \sigma, & \text { in } D_{\varepsilon},\end{cases}
$$

is the solution to (2.7). Thus $F\left(-\left.u_{\varepsilon}\right|_{\partial D_{\varepsilon}}+\left(1 /\left|\partial D_{\varepsilon}\right|\right) \int_{\partial D_{\varepsilon}} u_{\varepsilon} \mathrm{d} \sigma\right)=-\left.\frac{\partial u_{0}}{\partial \nu}\right|_{\partial D_{\varepsilon}}$. 
Altogether we obtain the following lemma from [11]:

Lemma 2.1. With $L_{\varepsilon}, L_{\varepsilon}^{*}$ and $F_{\varepsilon}$ defined by (2.4), (2.6) and (2.8), respectively, the difference of the Neumann-to-Dirichlet maps can be factorized as

$$
\Lambda_{\varepsilon}-\Lambda_{0}=L_{\varepsilon} F_{\varepsilon} L_{\varepsilon}^{*}
$$

Moreover, we find that the factorization yields the following mapping sequence:

$$
H_{\diamond}^{-1 / 2}(\partial \Omega) \stackrel{L_{\varepsilon}^{*}}{\longrightarrow} H_{\diamond}^{1 / 2}\left(\partial D_{\varepsilon}\right) \stackrel{F_{\varepsilon}}{\longrightarrow} H_{\diamond}^{-1 / 2}\left(\partial D_{\varepsilon}\right) \stackrel{L_{\varepsilon}}{\longrightarrow} H_{\diamond}^{1 / 2}(\partial \Omega)
$$

with

$$
f \stackrel{L_{\varepsilon}^{*}}{\longmapsto}-\left.u_{\varepsilon}\right|_{\partial D_{\varepsilon}}+\left(1 /\left|\partial D_{\varepsilon}\right|\right) \int_{\partial D_{\varepsilon}} u_{\varepsilon} \mathrm{d} \sigma \stackrel{F_{\varepsilon}}{\longmapsto}-\left.\left.\frac{\partial u_{0}}{\partial \nu}\right|_{\partial D_{\varepsilon}} \stackrel{L_{\varepsilon}}{\longmapsto}\left(u_{\varepsilon}-u_{0}\right)\right|_{\partial \Omega} .
$$

\section{Surface potentials}

Throughout we denote by $|x|$ the Euclidean norm of a point $x \in \mathbb{R}^{d}$, by $(x, y)$ the scalar product of two vectors $x, y \in \mathbb{R}^{d}$, and by $\omega_{d}$ the area of the $(d-1)$-dimensional unit sphere. The function

$$
\Phi(x-y):= \begin{cases}-\frac{1}{2 \pi} \log |x-y|, & \text { for } d=2, \\ \frac{1}{(d-2) \omega_{d}}|x-y|^{2-d}, & \text { for } d \geq 3,\end{cases}
$$

is called a fundamental solution for the Laplace equation.

Let $N$ denote the Neumann function for $\Delta$ in $\Omega$, i.e. for all $y \in \Omega, N(\cdot, y)$ is the unique solution to

$$
\begin{aligned}
\Delta_{x} N(x, y) & =-\delta_{y}, & & \text { for } x \in \Omega, \\
\frac{\partial N}{\partial \nu(x)}(x, y) & =-\frac{1}{|\partial \Omega|}, & & \text { for } x \in \partial \Omega,
\end{aligned}
$$

with the normalization $\int_{\partial \Omega} N(x, y) \mathrm{d} \sigma(x)=0$. Then $N$ is symmetric in its arguments in $(\Omega \times \Omega) \backslash \operatorname{diag}(\Omega \times \Omega)$, i.e.

$$
N(x, y)=N(y, x) \quad \text { for }(x, y) \in(\Omega \times \Omega) \backslash \operatorname{diag}(\Omega \times \Omega) ;
$$

cf. [6]. For each $y \in \Omega$ and $d \geq 2$, the Neumann function $N(x, y)$ has the form

$$
N(x, y)=\Phi(x-y)+R(x, y)
$$

where $R(\cdot, y)$ is the unique solution of the boundary value problem

$$
\begin{aligned}
\Delta_{x} R(x, y) & =0, & & \text { for } x \in \Omega, \\
\frac{\partial R}{\partial \nu(x)}(x, y) & =-\frac{1}{|\partial \Omega|}+\frac{1}{\omega_{d}} \frac{(x-y, \nu(x))}{|x-y|^{d}}, & & \text { for } x \in \partial \Omega,
\end{aligned}
$$

with $\int_{\partial \Omega} R(x, y) \mathrm{d} \sigma(x)=-\int_{\partial \Omega} \Phi(x-y) \mathrm{d} \sigma(x)$. Since $\Phi$ is symmetric, it follows that $R$ is symmetric in its arguments in $\Omega \times \Omega$. As a consequence, $R(x, \cdot)$ is a harmonic function on $\Omega$ for all $x \in \Omega$.

Given a bounded $C^{1, \alpha}$ domain $D \subset \mathbb{R}^{d}$, we denote the single layer potential and the double layer potential of a function $\phi \in C(\partial D)$ by

$$
\left(\mathcal{S}_{D} \phi\right)(x):=\int_{\partial D} \Phi(x-y) \phi(y) \mathrm{d} \sigma(y), \quad \text { for } x \in \mathbb{R}^{d},
$$


and

$$
\left(\mathcal{D}_{D} \phi\right)(x):=\int_{\partial D} \frac{\partial \Phi(x-y)}{\partial \nu(y)} \phi(y) \mathrm{d} \sigma(y), \quad \text { for } x \in \mathbb{R}^{d} \backslash \partial D .
$$

Then we have the following trace formulas (cf., e.g., [33):

$$
\begin{aligned}
\left.\frac{\partial}{\partial \nu} \mathcal{S}_{D} \phi\right|_{\partial D} ^{ \pm}(x) & =\left(\left(\mp \frac{1}{2} I+\mathcal{K}_{D}^{*}\right) \phi\right)(x), & & \text { for } x \in \partial D, \\
\left.\mathcal{D}_{D} \phi\right|_{\partial D} ^{ \pm}(x) & =\left(\left( \pm \frac{1}{2} I+\mathcal{K}_{D}\right) \phi\right)(x), & & \text { for } x \in \partial D,
\end{aligned}
$$

where $\mathcal{K}_{D}$ is defined by

$$
\left(\mathcal{K}_{D} \phi\right)(x):=\int_{\partial D} \frac{\partial \Phi(x-y)}{\partial \nu(y)} \phi(y) \mathrm{d} \sigma(y)=\frac{1}{\omega_{d}} \int_{\partial D} \frac{(x-y, \nu(y))}{|x-y|^{d}} \phi(y) \mathrm{d} \sigma(y)
$$

for $x \in \partial D$ and $\mathcal{K}_{D}^{*}$ is the adjoint of $\mathcal{K}_{D}$, i.e.

$$
\left(\mathcal{K}_{D}^{*} \phi\right)(x)=\int_{\partial D} \frac{\partial \Phi(x-y)}{\partial \nu(x)} \phi(y) \mathrm{d} \sigma(y)=\frac{1}{\omega_{d}} \int_{\partial D} \frac{(y-x, \nu(x))}{|x-y|^{d}} \phi(y) \mathrm{d} \sigma(y)
$$

for $x \in \partial D$.

$\mathcal{S}_{D}, \mathcal{D}_{D}, \mathcal{K}_{D}$ and $\mathcal{K}_{D}^{*}$ have continuous extensions

$$
\begin{aligned}
& \mathcal{S}_{D}: H^{-1 / 2}(\partial D) \rightarrow H_{l o c}^{1}\left(\mathbb{R}^{d}\right), \\
&\left.\mathcal{D}_{D}\right|_{D}: H^{1 / 2}(\partial D) \rightarrow H^{1}(D), \\
&\left.\mathcal{D}_{D}\right|_{\mathbb{R}^{d} \backslash \bar{D}}: H^{1 / 2}(\partial D) \rightarrow H_{l o c}^{1}\left(\mathbb{R}^{d} \backslash \bar{D}\right), \\
& \mathcal{K}_{D}: H^{1 / 2}(\partial D) \rightarrow H^{1 / 2}(\partial D), \\
& \mathcal{K}_{D}^{*}: H^{-1 / 2}(\partial D) \rightarrow H^{-1 / 2}(\partial D),
\end{aligned}
$$

and the jump formulas (3.2), (3.3) remain valid for these operators; cf. [21,35. Moreover, $\mathcal{K}_{D}$ as well as $\mathcal{K}_{D}^{*}$ is compact [36] and $-\frac{1}{2} I+\mathcal{K}_{D}$ has trivial nullspace in $H^{1 / 2}(\partial D)$ [6]. Hence, by the Fredholm alternative, $-\frac{1}{2} I+\mathcal{K}_{D}$ is invertible on $H^{1 / 2}(\partial D)$ and $-\frac{1}{2} I+\mathcal{K}_{D}^{*}$ is invertible on $H^{-1 / 2}(\partial D)$.

Since $\mathcal{K}_{D} 1=-\frac{1}{2}$, we get for each $\phi \in H^{-1 / 2}(\partial D)$,

$$
\int_{\partial D}\left(-\frac{1}{2} I+\mathcal{K}_{D}^{*}\right) \phi \mathrm{d} \sigma=-\int_{\partial D} \phi \mathrm{d} \sigma
$$

Thus, $-\frac{1}{2} I+\mathcal{K}_{D}^{*}$ maps $H_{\diamond}^{-1 / 2}(\partial D)$ onto $H_{\diamond}^{-1 / 2}(\partial D)$.

Next we consider the following modified surface potentials: Let $D$ be a bounded $C^{1, \alpha}$ domain compactly contained in $\Omega$. For a function $\phi \in C(\partial D)$ define

$$
\begin{array}{ll}
\left(\mathcal{S}_{D}^{N} \phi\right)(x):=\int_{\partial D} N(x, y) \phi(y) \mathrm{d} \sigma(y), & \text { for } x \in \Omega, \\
\left(\mathcal{D}_{D}^{N} \phi\right)(x):=\int_{\partial D} \frac{\partial N(x, y)}{\partial \nu(y)} \phi(y) \mathrm{d} \sigma(y), & \text { for } x \in \Omega \backslash \partial D .
\end{array}
$$


According to (3.1) we obtain the following trace formulas:

$$
\begin{aligned}
\left.\frac{\partial}{\partial \nu} \mathcal{S}_{D}^{N} \phi\right|_{\partial D} ^{ \pm}(x) & =\left(\left(\mp \frac{1}{2} I+\mathcal{K}_{D}^{*}\right) \phi\right)(x)+\int_{\partial D} \frac{\partial R(x, y)}{\partial \nu(x)} \phi(y) \mathrm{d} \sigma(y), \\
\left.\mathcal{D}_{D}^{N} \phi\right|_{\partial D} ^{ \pm}(x) & =\left(\left( \pm \frac{1}{2} I+\mathcal{K}_{D}\right) \phi\right)(x)+\int_{\partial D} \frac{\partial R(x, y)}{\partial \nu(y)} \phi(y) \mathrm{d} \sigma(y),
\end{aligned}
$$

for $x \in \partial D$. Define

$$
\left(\mathcal{R}_{D} \phi\right)(x):=\int_{\partial D} \frac{\partial R(x, y)}{\partial \nu(y)} \phi(y) \mathrm{d} \sigma(y), \quad \text { for } x \in \partial D,
$$

and let

$$
\mathcal{K}_{D}^{N} \phi:=\mathcal{K}_{D} \phi+\mathcal{R}_{D} \phi
$$

Then we obtain

$$
\begin{array}{rlrl}
\left.\frac{\partial}{\partial \nu} \mathcal{S}_{D}^{N} \phi\right|_{\partial D} ^{ \pm}(x) & =\left(\left(\mp \frac{1}{2} I+\left(\mathcal{K}_{D}^{N}\right)^{*}\right) \phi\right)(x), & & \text { for } x \in \partial D, \\
\left.\mathcal{D}_{D}^{N} \phi\right|_{\partial D} ^{ \pm}(x) & =\left(\left( \pm \frac{1}{2} I+\mathcal{K}_{D}^{N}\right) \phi\right)(x), & \text { for } x \in \partial D
\end{array}
$$

where $\left(\mathcal{K}_{D}^{N}\right)^{*}$ is the adjoint of $\mathcal{K}_{D}^{N}$.

Recalling (3.1) and the mapping properties of the boundary integral operators above we find that the operators

$$
\begin{gathered}
\mathcal{S}_{D}^{N}: H^{-1 / 2}(\partial D) \rightarrow H^{1}(\Omega), \\
\left.\mathcal{D}_{D}^{N}\right|_{D}: H^{1 / 2}(\partial D) \rightarrow H^{1}(D), \\
\left.\mathcal{D}_{D}^{N}\right|_{\Omega \backslash \bar{D}}: H^{1 / 2}(\partial D) \rightarrow H^{1}(\Omega \backslash \bar{D})
\end{gathered}
$$

are continuous and the jump relations (3.5), (3.6) remain valid for these extensions. The kernel of the integral operator $\mathcal{R}_{D}$ is continuous, so

$$
\mathcal{R}_{D}: H^{1 / 2}(\partial D) \rightarrow H^{1 / 2}(\partial D)
$$

and the corresponding dual operator

$$
\mathcal{R}_{D}^{*}: H^{-1 / 2}(\partial D) \rightarrow H^{-1 / 2}(\partial D)
$$

are compact. Therefore the operators

$$
\begin{gathered}
\mathcal{K}_{D}^{N}: H^{1 / 2}(\partial D) \rightarrow H^{1 / 2}(\partial D), \\
\left(\mathcal{K}_{D}^{N}\right)^{*}: H^{-1 / 2}(\partial D) \rightarrow H^{-1 / 2}(\partial D)
\end{gathered}
$$

are compact, too.

Lemma 3.1. The operators $-\frac{1}{2} I+\mathcal{K}_{D}^{N}$ and $-\frac{1}{2} I+\left(\mathcal{K}_{D}^{N}\right)^{*}$ have trivial nullspace in $H^{1 / 2}(\partial D)$ and $H^{-1 / 2}(\partial D)$, respectively.

Proof. Let $\phi \in H^{-1 / 2}(\partial D)$ be a solution to the equation $\left(-\frac{1}{2} I+\left(\mathcal{K}_{D}^{N}\right)^{*}\right) \phi=0$ and define $v:=\mathcal{S}_{D}^{N} \phi$. Then by (3.5)

$$
\left.\frac{\partial v}{\partial \nu}\right|_{\partial D} ^{+}=\left(-\frac{1}{2} I+\left(\mathcal{K}_{D}^{N}\right)^{*}\right) \phi=0
$$

and $v$ is a solution to the Neumann problem

$$
\Delta v=0 \quad \text { in } \Omega \backslash \bar{D},\left.\quad \frac{\partial v}{\partial \nu}\right|_{\partial \Omega}=C,\left.\quad \frac{\partial v}{\partial \nu}\right|_{\partial D}=0,
$$


where $C:=-\frac{1}{|\partial \Omega|} \int_{\partial D} \phi \mathrm{d} \sigma$ is constant. From the Divergence Theorem we obtain $C=0$. Thus we find that $v$ is constant in $\Omega \backslash \bar{D}$ and therefore on $\partial D$. Since $\Delta v=0$ in $D$ we obtain that $v$ is constant in $D$. From (3.5) we see that $\phi=$ $-\left.\frac{\partial v}{\partial \nu}\right|_{\partial D} ^{+}+\left.\frac{\partial v}{\partial \nu}\right|_{\partial D} ^{-}=0$. Hence, $\operatorname{ker}\left(-\frac{1}{2} I+\left(\mathcal{K}_{D}^{N}\right)^{*}\right)=\{0\}$.

By the Fredholm alternative it also follows that $\operatorname{ker}\left(-\frac{1}{2} I+\mathcal{K}_{D}^{N}\right)=\{0\}$ in $H^{1 / 2}(\partial D)$.

Applying the Fredholm alternative and Lemma 3.1 we find that $-\frac{1}{2} I+\mathcal{K}_{D}^{N}$ is invertible on $H^{1 / 2}(\partial D)$ and $-\frac{1}{2} I+\left(\mathcal{K}_{D}^{N}\right)^{*}$ is invertible on $H^{-1 / 2}(\partial D)$.

Since, for all $x \in \Omega, R(x, \cdot)$ is harmonic in $D$, it follows that

$$
\mathcal{K}_{D}^{N} 1=\mathcal{K}_{D} 1+\mathcal{R}_{D} 1=\mathcal{K}_{D} 1+\int_{\partial D} \frac{\partial R(\cdot, y)}{\partial \nu(y)} \mathrm{d} \sigma(y)=-\frac{1}{2},
$$

and we get as above that $-\frac{1}{2} I+\left(\mathcal{K}_{D}^{N}\right)^{*}$ maps $H_{\diamond}^{-1 / 2}(\partial D)$ onto $H_{\diamond}^{-1 / 2}(\partial D)$. Also as a consequence of this harmonicity, we find that the subspace of constant functions in $H^{1 / 2}(\partial D)$ is contained in the nullspace of $\mathcal{R}_{D}$. Moreover, applying the harmonicity of $R(\cdot, y)$ for all $y \in \Omega$, we see that $\mathcal{R}_{D}^{*}$ maps $H^{-1 / 2}(\partial D)$ into $H_{\diamond}^{-1 / 2}(\partial D)$. Therefore, in the following we may consider $\mathcal{R}_{D}$ and $\mathcal{R}_{D}^{*}$ as dual operators from $H_{\diamond}^{1 / 2}(\partial D)$ to $H^{1 / 2}(\partial D)$ and $H^{-1 / 2}(\partial D)$ to $H_{\diamond}^{-1 / 2}(\partial D)$, respectively.

\section{First estimates}

In the following sections we often have to deal with changes of coordinates. Therefore we introduce some notation: Given $\phi \in L^{2}\left(\partial D_{\varepsilon}\right)$ and $\psi \in L^{2}(\partial B)$ we define $\hat{\phi},(\phi)^{\wedge} \in L^{2}(\partial B)$ and $\check{\psi},(\psi)^{\vee} \in L^{2}\left(\partial D_{\varepsilon}\right)$ by

$$
\begin{aligned}
(\phi)^{\wedge}(\xi):=\hat{\phi}(\xi):=\phi(\varepsilon \xi+z), & & \text { for a.e. } \xi \in \partial B, \\
(\psi)^{\vee}(x):=\check{\psi}(x):=\psi\left(\frac{x-z}{\varepsilon}\right), & & \text { for a.e. } x \in \partial D_{\varepsilon},
\end{aligned}
$$

respectively. The same notation will also be used for functions in $H^{1 / 2}\left(\partial D_{\varepsilon}\right)$ or $H^{-1 / 2}\left(\partial D_{\varepsilon}\right)$ and $H^{1 / 2}(\partial B)$ or $H^{-1 / 2}(\partial B)$, respectively. This makes sense, since the corresponding Sobolev spaces on $\mathbb{R}^{d-1}$ are invariant under such regular changes of coordinates (cf. 35]). Moreover, we apply the notation to functions in $H^{1}\left(D_{\varepsilon}\right)$ and $H^{1}(B)$ in the same way.

In our estimates we shall use a generic constant $C$.

For bounded $C^{1, \alpha}$ domains $D \subset \mathbb{R}^{d}$ we use the following norm on $H^{1 / 2}(\partial D)$ (cf., e.g., [27]):

$$
\|\phi\|_{H^{1 / 2}(\partial D)}:=\inf _{\substack{\left.u \in H^{1}(D) \\ u\right|_{\partial D}=\phi}}\|u\|_{H^{1}(D)}, \quad \text { for all } \phi \in H^{1 / 2}(\partial D) .
$$

The dual space $H^{-1 / 2}(\partial D)$ shall be equipped with the corresponding dual norm:

$$
\|\psi\|_{H^{-1 / 2}(\partial D)}:=\sup _{\substack{\phi \in H^{1 / 2}(\partial D) \\ \phi \neq 0}} \frac{\left|\langle\psi, \phi\rangle_{\partial D}\right|}{\|\phi\|_{H^{1 / 2}(\partial D)}}, \quad \text { for all } \psi \in H^{-1 / 2}(\partial D),
$$

where $\langle\cdot, \cdot\rangle_{\partial D}$ denotes the duality pairing between $H^{1 / 2}(\partial D)$ and $H^{-1 / 2}(\partial D)$.

The following lemma examines the scaling properties of these norms under changes of coordinates as in (4.1): 
Lemma 4.1. Suppose $0<\epsilon \leq 1$. Then there exist constants $c$ and $C$ independent of $\varepsilon$ such that for each $\phi \in H_{\diamond}^{1 / 2}\left(\partial D_{\varepsilon}\right)$ and $\psi \in H_{\diamond}^{-1 / 2}\left(\partial D_{\varepsilon}\right)$,

$$
\begin{aligned}
\varepsilon^{\frac{d-2}{2}} c\|\hat{\phi}\|_{H^{1 / 2}(\partial B)} & \leq\|\phi\|_{H^{1 / 2}\left(\partial D_{\varepsilon}\right)} \leq \varepsilon^{\frac{d-2}{2}}\|\hat{\phi}\|_{H^{1 / 2}(\partial B)}, \\
\varepsilon^{\frac{d}{2}}\|\hat{\psi}\|_{H^{-1 / 2}(\partial B)} & \leq\|\psi\|_{H^{-1 / 2}\left(\partial D_{\varepsilon}\right)} \leq \varepsilon^{\frac{d}{2}} C\|\hat{\psi}\|_{H^{-1 / 2}(\partial B)} .
\end{aligned}
$$

Proof. Let $\phi \in H_{\diamond}^{1 / 2}\left(\partial D_{\varepsilon}\right)$ and $\psi \in H_{\diamond}^{-1 / 2}\left(\partial D_{\varepsilon}\right)$. By change of coordinates, $\xi:=$ $\frac{x-z}{\varepsilon}$, we observe that for all $u \in H_{\diamond}^{1}\left(D_{\varepsilon}\right)$,

$$
\begin{aligned}
\|u\|_{H^{1}\left(D_{\varepsilon}\right)}^{2} & =\int_{D_{\varepsilon}}\left(|u(x)|^{2}+\left|\nabla_{x} u(x)\right|^{2}\right) \mathrm{d} x \\
& =\varepsilon^{d} \int_{B}\left(|u(\varepsilon \xi+z)|^{2}+\frac{1}{\varepsilon^{2}}\left|\nabla_{\xi} u(\varepsilon \xi+z)\right|^{2}\right) \mathrm{d} \xi \\
& =\varepsilon^{d} \int_{B}\left(|\hat{u}(\xi)|^{2}+\frac{1}{\varepsilon^{2}}\left|\nabla_{\xi} \hat{u}(\xi)\right|^{2}\right) \mathrm{d} \xi .
\end{aligned}
$$

Thus,

$$
\|u\|_{H^{1}\left(D_{\varepsilon}\right)}^{2} \leq \varepsilon^{d-2}\|\hat{u}\|_{H^{1}(B)}^{2},
$$

since we assumed that $0<\varepsilon \leq 1$, and therefore

$$
\|\phi\|_{H^{1 / 2}\left(\partial D_{\varepsilon}\right)} \leq \varepsilon^{\frac{d-2}{2}}\|\hat{\phi}\|_{H^{1 / 2}(\partial B)} .
$$

The Poincaré inequality (cf. [22, Chapter IV, Section 7, Proposition 2]), implies that there exists a constant $c$ independent of $\varepsilon$ such that for all $u \in H_{\diamond}^{1}\left(D_{\varepsilon}\right)$,

$$
\varepsilon^{d-2} c\|\hat{u}\|_{H^{1}(B)}^{2} \leq \varepsilon^{d-2}\left\|\nabla_{\xi} \hat{u}\right\|_{L^{2}(B)}^{2}=\left\|\nabla_{x} u\right\|_{L^{2}\left(D_{\varepsilon}\right)}^{2} \leq\|u\|_{H^{1}\left(D_{\varepsilon}\right)}^{2} .
$$

Hence,

$$
\varepsilon^{\frac{d-2}{2}} c\|\hat{\phi}\|_{H^{1 / 2}(\partial B)} \leq\|\phi\|_{H^{1 / 2}\left(\partial D_{\varepsilon}\right)} .
$$

For the dual norm we obtain by change of coordinates, applying (4.2),

$$
\begin{aligned}
\|\psi\|_{H^{-1 / 2}\left(\partial D_{\varepsilon}\right)} & =\sup _{\substack{\phi \in H^{1 / 2}\left(\partial D_{\varepsilon}\right) \\
\phi \neq 0}} \frac{\left|\langle\psi, \phi\rangle_{\partial D_{\varepsilon} \mid}\right|}{\|\phi\|_{H^{1 / 2}\left(\partial D_{\varepsilon}\right)}} \geq \sup _{\substack{\phi \in H^{1 / 2}\left(\partial D_{\varepsilon}\right) \\
\phi \neq 0}} \frac{\varepsilon^{d-1}\left|\langle\hat{\psi}, \hat{\phi}\rangle_{\partial B}\right|}{\varepsilon^{\frac{d-2}{2}}\|\hat{\phi}\|_{H^{1 / 2}(\partial B)}} \\
& =\varepsilon^{\frac{d}{2}} \sup _{\substack{\hat{\phi} \in H^{1 / 2}(\partial B) \\
\hat{\phi} \neq 0}} \frac{\left|\langle\hat{\psi}, \hat{\phi}\rangle_{\partial B}\right|}{\|\hat{\phi}\|_{H^{1 / 2}(\partial B)}}=\varepsilon^{\frac{d}{2}}\|\hat{\psi}\|_{H^{-1 / 2}(\partial B)}
\end{aligned}
$$

and in the same way

$$
\begin{aligned}
& \|\psi\|_{H^{-1 / 2}\left(\partial D_{\varepsilon}\right)} \leq \sup _{\substack{\phi \in H^{1 / 2}\left(\partial D_{\varepsilon}\right) \\
\phi \neq 0}} \frac{\varepsilon^{d-1}\left|\langle\hat{\psi}, \hat{\phi}\rangle_{\partial B}\right|}{\varepsilon^{\frac{d-2}{2}} c\|\hat{\phi}\|_{H^{1 / 2}(\partial B)}} \\
& =c^{-1} \varepsilon^{\frac{d}{2}} \sup _{\substack{\hat{\phi} \in H^{1 / 2}(\partial B) \\
\hat{\phi} \neq 0}} \frac{\left|\langle\hat{\psi}, \hat{\phi}\rangle_{\partial B}\right|}{\|\hat{\phi}\|_{H^{1 / 2}(\partial B)}}=\varepsilon^{\frac{d}{2}} C\|\hat{\psi}\|_{H^{-1 / 2}(\partial B)} .
\end{aligned}
$$

Here we put $C:=c^{-1}$.

In the next lemma we investigate the scaling properties of the integral operators $\mathcal{K}_{D_{\varepsilon}}$ and $\mathcal{K}_{D_{\varepsilon}}^{*}$ : 
Lemma 4.2. Let $\phi \in H^{1 / 2}\left(\partial D_{\varepsilon}\right)$ and $\psi \in H^{-1 / 2}\left(\partial D_{\varepsilon}\right)$. Then

$$
\mathcal{K}_{D_{\varepsilon}} \phi=\left(\mathcal{K}_{B} \hat{\phi}\right)^{\vee} \quad \text { and } \quad \mathcal{K}_{D_{\varepsilon}}^{*} \psi=\left(\mathcal{K}_{B}^{*} \hat{\psi}\right)^{\vee} .
$$

Proof. By change of variables, $\xi:=\frac{x-z}{\varepsilon}$ and $\eta:=\frac{y-z}{\varepsilon}$, we see that for a.e. $x \in \partial D_{\varepsilon}$,

$$
\begin{aligned}
\left(\mathcal{K}_{D_{\varepsilon}} \phi\right)(x) & =\frac{1}{\omega_{d}} \int_{\partial D_{\varepsilon}} \frac{(x-y, \nu(y))}{|x-y|^{d}} \phi(y) \mathrm{d} \sigma(y) \\
& =\frac{1}{\omega_{d}} \int_{\partial B} \frac{(\varepsilon \xi-\varepsilon \eta, \nu(\eta))}{|\varepsilon \xi-\varepsilon \eta|^{d}} \phi(\varepsilon \eta+z) \varepsilon^{d-1} \mathrm{~d} \sigma(\eta) \\
& =\frac{1}{\omega_{d}} \int_{\partial B} \frac{(\xi-\eta, \nu(\eta))}{|\xi-\eta|^{d}} \hat{\phi}(\eta) \mathrm{d} \sigma(\eta) \\
& =\left(\mathcal{K}_{B} \hat{\phi}\right)(\xi) .
\end{aligned}
$$

The second identity follows in the same way.

Next we will estimate the norm of the operator $\mathcal{R}_{D_{\varepsilon}} \in \mathcal{L}\left(H_{\diamond}^{1 / 2}\left(\partial D_{\varepsilon}\right), H^{1 / 2}\left(\partial D_{\varepsilon}\right)\right)$.

Lemma 4.3. There exists a constant $C$ independent of $\varepsilon$ such that for each $\phi \in$ $H_{\diamond}^{1 / 2}\left(\partial D_{\varepsilon}\right)$

$$
\left\|\mathcal{R}_{D_{\varepsilon}} \phi\right\|_{H^{1 / 2}\left(\partial D_{\varepsilon}\right)} \leq \varepsilon^{d} C\|\phi\|_{H^{1 / 2}\left(\partial D_{\varepsilon}\right)} .
$$

Proof. Let $\phi \in H_{\diamond}^{1 / 2}\left(\partial D_{\varepsilon}\right)$. By $\widetilde{\mathcal{R}}_{D_{\varepsilon}} \phi$ we denote the extension of $\mathcal{R}_{D_{\varepsilon}} \phi$ to $H^{1}\left(D_{\varepsilon}\right)$ which is obtained canonically via (3.4). Then, since $R$ and $\nabla_{x} R$ are uniformly bounded near the centre of the inclusion,

$$
\begin{aligned}
& \left\|\mathcal{R}_{D_{\varepsilon}} \phi\right\|_{H^{1 / 2}\left(\partial D_{\varepsilon}\right)}^{2}=\left(\inf _{\substack{\left.u \in H^{1}\left(D_{\varepsilon}\right) \\
u\right|_{\partial D_{\varepsilon}=\mathcal{R}_{D_{\varepsilon}} \phi}}}\|u\|_{H^{1}\left(D_{\varepsilon}\right)}\right)^{2} \leq\left\|\widetilde{\mathcal{R}}_{D_{\varepsilon}} \phi\right\|_{H^{1}\left(D_{\varepsilon}\right)}^{2} \\
& =\int_{D_{\varepsilon}}\left|\int_{\partial D_{\varepsilon}} \frac{\partial R(x, y)}{\partial \nu(y)} \phi(y) \mathrm{d} \sigma(y)\right|^{2} \mathrm{~d} x \\
& +\int_{D_{\varepsilon}}\left|\nabla_{x} \int_{\partial D_{\varepsilon}} \frac{\partial R(x, y)}{\partial \nu(y)} \phi(y) \mathrm{d} \sigma(y)\right|^{2} \mathrm{~d} x \\
& \leq \int_{D_{\varepsilon}}\left(\int _ { \partial D _ { \varepsilon } } \left(\left|\frac{\partial R(x, y)}{\partial \nu(y)}\right|^{2}\right.\right. \\
& \left.\left.+\left|\nabla_{x} \frac{\partial R(x, y)}{\partial \nu(y)}\right|^{2}\right) \mathrm{~d} \sigma(y) \int_{\partial D_{\varepsilon}}|\phi(y)|^{2} \mathrm{~d} \sigma(y)\right) \mathrm{d} x \\
& \leq \varepsilon^{d-1} C\|\phi\|_{L^{2}\left(\partial D_{\varepsilon}\right)}^{2} \int_{D_{\varepsilon}} 1 \mathrm{~d} x \leq \varepsilon^{2 d-1} C\|\phi\|_{L^{2}\left(\partial D_{\varepsilon}\right)}^{2}
\end{aligned}
$$

with a constant $C$ that is independent of $\varepsilon$. Moreover, applying the Sobolev Imbedding Theorem and Lemma 4.1, we find

$$
\|\phi\|_{L^{2}\left(\partial D_{\varepsilon}\right)}^{2}=\varepsilon^{d-1}\|\hat{\phi}\|_{L^{2}(\partial B)}^{2} \leq \varepsilon^{d-1} C\|\hat{\phi}\|_{H^{1 / 2}(\partial B)}^{2} \leq \varepsilon C\|\phi\|_{H^{1 / 2}\left(\partial D_{\varepsilon}\right)}^{2}
$$

with a constant $C$ that is independent of $\varepsilon$. Combining these two estimates yields the assertion. 
Therefore, we have $\mathcal{R}_{D_{\varepsilon}}=\mathcal{O}\left(\varepsilon^{d}\right)$ and

$$
-\frac{1}{2} I+\mathcal{K}_{D_{\varepsilon}}^{N}=-\frac{1}{2} I+\mathcal{K}_{D_{\varepsilon}}+\mathcal{R}_{D_{\varepsilon}}=-\frac{1}{2} I+\mathcal{K}_{D_{\varepsilon}}+\mathcal{O}\left(\varepsilon^{d}\right)
$$

in $\mathcal{L}\left(H_{\diamond}^{1 / 2}\left(\partial D_{\varepsilon}\right), H^{1 / 2}\left(\partial D_{\varepsilon}\right)\right)$, as $\varepsilon \rightarrow 0$, where the remainder estimate $\mathcal{O}\left(\varepsilon^{d}\right)$ is in terms of the operator norm in $\mathcal{L}\left(H_{\diamond}^{1 / 2}\left(\partial D_{\varepsilon}\right), H^{1 / 2}\left(\partial D_{\varepsilon}\right)\right)$. By duality we find that also $\mathcal{R}_{D_{\varepsilon}}^{*}=\mathcal{O}\left(\varepsilon^{d}\right)$ and

$$
-\frac{1}{2} I+\left(\mathcal{K}_{D_{\varepsilon}}^{N}\right)^{*}=-\frac{1}{2} I+\mathcal{K}_{D_{\varepsilon}}^{*}+\mathcal{R}_{D_{\varepsilon}}^{*}=-\frac{1}{2} I+\mathcal{K}_{D_{\varepsilon}}^{*}+\mathcal{O}\left(\varepsilon^{d}\right)
$$

in $\mathcal{L}\left(H^{-1 / 2}\left(\partial D_{\varepsilon}\right), H_{\diamond}^{-1 / 2}\left(\partial D_{\varepsilon}\right)\right)$. For $\mathcal{L}\left(H_{\diamond}^{-1 / 2}\left(\partial D_{\varepsilon}\right), H_{\diamond}^{-1 / 2}\left(\partial D_{\varepsilon}\right)\right)$, the latter result also holds.

In the following we consider $-\frac{1}{2} I+\mathcal{K}_{D_{\varepsilon}}^{*}$ and $-\frac{1}{2} I+\left(\mathcal{K}_{D_{\varepsilon}}^{N}\right)^{*}$ as operators in $\mathcal{L}\left(H_{\diamond}^{-1 / 2}\left(\partial D_{\varepsilon}\right), H_{\diamond}^{-1 / 2}\left(\partial D_{\varepsilon}\right)\right)$. From Lemma 4.2 we obtain for all $\psi \in H_{\diamond}^{-1 / 2}\left(\partial D_{\varepsilon}\right)$ that

$$
\left(-\frac{1}{2} I+\mathcal{K}_{D_{\varepsilon}}^{*}\right) \psi=\left(\left(-\frac{1}{2} I+\mathcal{K}_{B}^{*}\right) \hat{\psi}\right)^{\vee}
$$

and

$$
\left(-\frac{1}{2} I+\mathcal{K}_{D_{\varepsilon}}^{*}\right)^{-1} \psi=\left(\left(-\frac{1}{2} I+\mathcal{K}_{B}^{*}\right)^{-1} \hat{\psi}\right)^{\vee}
$$

Therefore, applying Lemma 4.1 and Lemma 4.2, we find that

$$
\begin{aligned}
& \left\|\left(-\frac{1}{2} I+\mathcal{K}_{D_{\varepsilon}}^{*}\right)^{-1}\right\|_{\partial D_{\varepsilon}}=\sup _{\substack{\psi \in H_{\diamond}^{-1 / 2}\left(\partial D_{\varepsilon}\right) \\
\psi \neq 0}} \frac{\left\|\left(-\frac{1}{2} I+\mathcal{K}_{D_{\varepsilon}}^{*}\right)^{-1} \psi\right\|_{H^{-1 / 2}\left(\partial D_{\varepsilon}\right)}}{\|\psi\|_{H^{-1 / 2}\left(\partial D_{\varepsilon}\right)}} \\
& \leq \sup _{\substack{\psi \in H_{\diamond}^{-1 / 2}\left(\partial D_{\varepsilon}\right) \\
\psi \neq 0}} \frac{\varepsilon^{\frac{d}{2}} C\left\|\left(\left(-\frac{1}{2} I+\mathcal{K}_{D_{\varepsilon}}^{*}\right)^{-1} \psi\right)^{\wedge}\right\|_{H^{-1 / 2}(\partial B)}}{\varepsilon^{\frac{d}{2}}\|\hat{\psi}\|_{H^{-1 / 2}(\partial B)}} \\
& =C \sup _{\substack{\hat{\psi} \in H_{\diamond}^{-1 / 2}(\partial B) \\
\hat{\psi} \neq 0}} \frac{\left\|\left(-\frac{1}{2} I+\mathcal{K}_{B}^{*}\right)^{-1} \hat{\psi}\right\|_{H^{-1 / 2}(\partial B)}}{\|\hat{\psi}\|_{H^{-1 / 2}(\partial B)}} \\
& =C\left\|\left(-\frac{1}{2} I+\mathcal{K}_{B}^{*}\right)^{-1}\right\|_{\partial B},
\end{aligned}
$$

where $\|\cdot\|_{\partial D_{\varepsilon}}$ and $\|\cdot\|_{\partial B}$ denote the operator norm on $\mathcal{L}\left(H_{\diamond}^{-1 / 2}\left(\partial D_{\varepsilon}\right), H_{\diamond}^{-1 / 2}\left(\partial D_{\varepsilon}\right)\right)$ and $\mathcal{L}\left(H_{\diamond}^{-1 / 2}(\partial B), H_{\diamond}^{-1 / 2}(\partial B)\right)$, respectively, and the constant $C$ is independent of $\varepsilon$.

From this estimate it follows that $\left\|\left(-\frac{1}{2} I+\mathcal{K}_{D_{\varepsilon}}^{*}\right)^{-1}\right\|_{\partial D_{\varepsilon}} \leq C$, with a constant $C$ that is independent of $\varepsilon$. Together with (4.4) and a Neumann series argument (cf., e.g., 33]), we thus obtain

$$
\left(-\frac{1}{2} I+\left(\mathcal{K}_{D_{\varepsilon}}^{N}\right)^{*}\right)^{-1}=\left(-\frac{1}{2} I+\mathcal{K}_{D_{\varepsilon}}^{*}\right)^{-1}+\mathcal{P}_{D_{\varepsilon}}
$$

with $\mathcal{P}_{D_{\varepsilon}}=\mathcal{O}\left(\varepsilon^{d}\right)$ in $\mathcal{L}\left(H_{\diamond}^{-1 / 2}\left(\partial D_{\varepsilon}\right), H_{\diamond}^{-1 / 2}\left(\partial D_{\varepsilon}\right)\right)$. 


\section{Asymptotic EXPANSION}

Now we consider the boundary value problem (2.3) and the operator $L_{\varepsilon}$ from (2.4). For $\phi \in H_{\diamond}^{-1 / 2}\left(\partial D_{\varepsilon}\right)$ we define $v_{\varepsilon} \in H_{\diamond, \partial \Omega}^{1}\left(\Omega \backslash \overline{D_{\varepsilon}}\right)$ by

$$
v_{\varepsilon}:=\mathcal{S}_{D_{\varepsilon}}^{N}\left(-\frac{1}{2} I+\left(\mathcal{K}_{D_{\varepsilon}}^{N}\right)^{*}\right)^{-1} \phi
$$

Then $v_{\varepsilon}$ is a solution to (2.3) and on $\partial \Omega$ we have

$$
\begin{aligned}
\left.v_{\varepsilon}\right|_{\partial \Omega}= & \int_{\partial D_{\varepsilon}} N(\cdot, y)\left(\left(-\frac{1}{2} I+\left(\mathcal{K}_{D_{\varepsilon}}^{N}\right)^{*}\right)^{-1} \phi\right)(y) \mathrm{d} \sigma(y) \\
= & \int_{\partial D_{\varepsilon}} N(\cdot, y)\left(\left(-\frac{1}{2} I+\mathcal{K}_{D_{\varepsilon}}^{*}\right)^{-1} \phi\right)(y) \mathrm{d} \sigma(y) \\
& +\int_{\partial D_{\varepsilon}} N(\cdot, y)\left(\mathcal{P}_{D_{\varepsilon}} \phi\right)(y) \mathrm{d} \sigma(y) .
\end{aligned}
$$

Recalling (4.3) we can estimate the last term on the right hand side as follows:

$$
\begin{gathered}
\left\|\int_{\partial D_{\varepsilon}} N(\cdot, y)\left(\mathcal{P}_{D_{\varepsilon}} \phi\right)(y) \mathrm{d} \sigma(y)\right\|_{H^{1 / 2}(\partial \Omega)}^{2} \\
\leq\left\|\int_{\partial D_{\varepsilon}} N(\cdot, y)\left(\mathcal{P}_{D_{\varepsilon}} \phi\right)(y) \mathrm{d} \sigma(y)\right\|_{H^{1}(\partial \Omega)}^{2} \\
\leq C\left(\max _{x \in \partial \Omega}\left|\int_{\partial D_{\varepsilon}} N(x, y)\left(\mathcal{P}_{D_{\varepsilon}} \phi\right)(y) \mathrm{d} \sigma(y)\right|^{2}\right. \\
\left.\quad+\max _{x \in \partial \Omega}\left|\int_{\partial D_{\varepsilon}} \nabla_{x} N(x, y)\left(\mathcal{P}_{D_{\varepsilon}} \phi\right)(y) \mathrm{d} \sigma(y)\right|^{2}\right) \\
\leq C\left\|\mathcal{P}_{D_{\varepsilon}} \phi\right\|_{H^{-1 / 2}\left(\partial D_{\epsilon}\right)}^{2}\left(\max _{x \in \partial \Omega}\|N(x, \cdot)\|_{H^{1 / 2}\left(\partial D_{\epsilon}\right)}^{2}\right. \\
\left.\quad+\max _{1 \leq j \leq d x \in \partial \Omega}\left\|\frac{\partial N}{\partial x_{j}}(x, \cdot)\right\|_{H^{1 / 2}\left(\partial D_{\epsilon}\right)}^{2}\right) \\
\leq C \epsilon^{d-1} \epsilon^{2 d}\|\phi\|_{H^{-1 / 2}\left(\partial D_{\epsilon}\right)}^{2} \leq C \epsilon^{4 d-1}\|\hat{\phi}\|_{H^{-1 / 2}(\partial B)}^{2},
\end{gathered}
$$

where the constant $C$ is independent of $\varepsilon$ and $\phi$.

Using the Taylor expansion we obtain for $x \in \partial \Omega, z \in \Omega$ and $\eta \in \partial B$ as $\varepsilon \rightarrow 0$,

$$
N(x, \varepsilon \eta+z)=\sum_{|j|=0}^{\infty} \frac{1}{j !} \varepsilon^{|j|} \partial_{y}^{j} N(x, z) \eta^{j},
$$

where $j=\left(j_{1}, \ldots, j_{d}\right)$ is a multi-index (cf. [35, p. 61]). Thus, recalling Lemma 4.2 and the fact that $-\frac{1}{2} I+\left(\mathcal{K}_{D_{\varepsilon}}^{N}\right)^{*}$ maps $H_{\diamond}^{-1 / 2}\left(\partial D_{\varepsilon}\right)$ into $H_{\diamond}^{-1 / 2}\left(\partial D_{\varepsilon}\right)$, we obtain the 
following asymptotic formula:

$$
\begin{aligned}
\left.v_{\varepsilon}\right|_{\partial \Omega}= & \varepsilon^{d-1} \int_{\partial B} N(\cdot, \varepsilon \eta+z)\left(\left(-\frac{1}{2} I+\mathcal{K}_{D_{\varepsilon}}^{*}\right)^{-1} \phi\right)(\varepsilon \eta+z) \mathrm{d} \sigma(\eta) \\
& +\mathcal{O}\left(\varepsilon^{2 d-\frac{1}{2}}\right) \\
= & \varepsilon^{d} \nabla_{y} N(\cdot, z) \cdot \int_{\partial B} \eta\left(\left(-\frac{1}{2} I+\mathcal{K}_{B}^{*}\right)^{-1} \hat{\phi}\right)(\eta) \mathrm{d} \sigma(\eta)+\mathcal{O}\left(\varepsilon^{d+1}\right) .
\end{aligned}
$$

The last term is bounded by $C \varepsilon^{d+1}\|\hat{\phi}\|_{H^{-1 / 2}(\partial B)}$ in $H_{\diamond}^{1 / 2}(\partial \Omega)$, where the constant $C$ is independent of $\varepsilon$ and $\phi$.

Definition 5.1. Define

$$
\begin{aligned}
L: H_{\diamond}^{-1 / 2}(\partial B) \rightarrow & H_{\diamond}^{1 / 2}(\partial \Omega), \\
& L \varphi:=\nabla_{y} N(\cdot, z) \cdot \int_{\partial B} \eta\left(\left(-\frac{1}{2} I+\mathcal{K}_{B}^{*}\right)^{-1} \varphi\right)(\eta) \mathrm{d} \sigma(\eta) .
\end{aligned}
$$

Then $L$ is a bounded linear operator, and we have shown the following asymptotic formula:

Proposition 5.2. For all $\phi \in H_{\diamond}^{-1 / 2}\left(\partial D_{\varepsilon}\right)$,

$$
L_{\varepsilon} \phi=\varepsilon^{d} L \hat{\phi}+E_{L} \hat{\phi}
$$

as $\varepsilon \rightarrow 0$, where the operator $E_{L}$ is bounded by $C \varepsilon^{d+1}$ in the norm of $\mathcal{L}\left(H_{\diamond}^{-1 / 2}(\partial B), H_{\diamond}^{1 / 2}(\partial \Omega)\right)$, and the constant $C$ is independent of $\varepsilon$.

Remark 5.3. By duality, in $\mathcal{L}\left(H_{\diamond}^{-1 / 2}(\partial \Omega), H_{\diamond}^{1 / 2}(\partial B)\right)$, the adjoint operator $E_{L}^{*}$ is $\mathcal{O}\left(\varepsilon^{d+1}\right)$.

Next we return to the diffraction problem (2.7) and the operator $F_{\varepsilon}$ from (2.8). Given $\chi \in H_{\diamond}^{1 / 2}\left(\partial D_{\varepsilon}\right)$ we define

$$
w_{\varepsilon}:=\mathcal{D}_{D_{\varepsilon}}^{N} \chi
$$

Then $w_{\varepsilon}$ is a solution to (2.7) and from (3.6) we obtain

$$
\left.w_{\varepsilon}\right|_{\partial D_{\varepsilon}} ^{-}=\left(-\frac{1}{2} I+\mathcal{K}_{D_{\varepsilon}}^{N}\right) \chi
$$

For $\varphi \in H^{1 / 2}\left(\partial D_{\varepsilon}\right)$ we consider the interior Dirichlet problem

$$
\Delta w=0 \quad \text { in } D_{\varepsilon}, \quad w=\varphi \quad \text { on } \partial D_{\varepsilon},
$$

and the corresponding interior Dirichlet-to-Neumann operator,

$$
\Upsilon_{\varepsilon}: H^{1 / 2}\left(\partial D_{\varepsilon}\right) \rightarrow H_{\diamond}^{-1 / 2}\left(\partial D_{\varepsilon}\right), \quad \Upsilon_{\varepsilon} \varphi:=\left.\frac{\partial w}{\partial \nu}\right|_{\partial D_{\varepsilon}} .
$$

Since $w_{\varepsilon}$ solves the diffraction problem (2.7), we obtain

$$
\left.\frac{\partial w_{\varepsilon}}{\partial \nu}\right|_{\partial D_{\varepsilon}}=\Upsilon_{\varepsilon}\left(-\frac{1}{2} I+\mathcal{K}_{D_{\varepsilon}}^{N}\right) \chi
$$

We define the interior Dirichlet-to-Neumann operator $\Upsilon: H^{1 / 2}(\partial B) \rightarrow H_{\diamond}^{-1 / 2}(\partial B)$ on $\partial B$ in the same way as $\Upsilon_{\varepsilon}$. These Dirichlet-to-Neumann maps are bounded linear operators. Next we take a closer look at the scaling properties of $\Upsilon_{\varepsilon}$. 
Lemma 5.4. Let $\varphi \in H^{1 / 2}\left(\partial D_{\varepsilon}\right)$. Then

$$
\varepsilon^{-1}(\Upsilon \hat{\varphi})^{\vee}=\Upsilon_{\varepsilon} \varphi
$$

Proof. Suppose $w$ is a solution to (5.3). By change of variables, $\xi:=\frac{x-z}{\varepsilon}$, we find that $\hat{w}$ satisfies

$$
\begin{aligned}
\Delta_{\xi} \hat{w}(\xi) & =\varepsilon^{2} \Delta_{x} w(x)=0, & & \text { for a.e. } \xi \in B, \\
\frac{\partial \hat{w}}{\partial \nu(\xi)}(\xi) & =\varepsilon \frac{\partial w}{\partial \nu(x)}(x), & & \text { for a.e. } \xi \in \partial B, \\
\hat{w}(\xi) & =w(x), & & \text { for a.e. } \xi \in \partial B .
\end{aligned}
$$

Hence,

$$
\left(\left.\Upsilon \hat{w}\right|_{\partial B}\right)(\xi)=\frac{\partial \hat{w}}{\partial \nu(\xi)}(\xi)=\varepsilon \frac{\partial w}{\partial \nu(x)}(x)=\varepsilon\left(\left.\Upsilon_{\varepsilon} w\right|_{\partial D_{\varepsilon}}\right)(x),
$$

for a.e. $\xi \in \partial B$ and $x=\varepsilon \xi+z \in \partial D_{\varepsilon}$.

Lemma 5.5. There exists a constant $C$ independent of $\varepsilon$ such that for each $\varphi \in$ $H_{\diamond}^{1 / 2}\left(\partial D_{\varepsilon}\right)$

$$
\left\|\left(\Upsilon_{\varepsilon} \mathcal{R}_{D_{\varepsilon}} \varphi\right)^{\wedge}\right\|_{H^{-1 / 2}(\partial B)} \leq \varepsilon^{d-1} C\|\hat{\varphi}\|_{H^{1 / 2}(\partial B)} .
$$

Proof. Let $\varphi \in H_{\diamond}^{1 / 2}\left(\partial D_{\varepsilon}\right)$. Using Lemma 5.4 , the continuity of $\Upsilon$, Lemma 4.1 and Lemma 4.3 we obtain

$$
\begin{aligned}
\left\|\left(\Upsilon_{\varepsilon} \mathcal{R}_{D_{\varepsilon}} \varphi\right)^{\wedge}\right\|_{H^{-1 / 2}(\partial B)} & =\varepsilon^{-1}\left\|\Upsilon \widehat{\mathcal{R}_{D_{\varepsilon}} \varphi}\right\|_{H^{-1 / 2}(\partial B)} \\
& \leq \varepsilon^{-1} C\left\|\widehat{\mathcal{R}_{D_{\varepsilon}}}\right\|_{H^{1 / 2}(\partial B)} \\
& \leq \varepsilon^{-1} C \varepsilon^{\frac{2-d}{2}}\left\|\mathcal{R}_{D_{\varepsilon}} \varphi\right\|_{H^{1 / 2}\left(\partial D_{\varepsilon}\right)} \\
& \leq \varepsilon^{-\frac{d}{2}} C \varepsilon^{d}\|\varphi\|_{H^{1 / 2}\left(\partial D_{\varepsilon}\right)} \\
& \leq \varepsilon^{\frac{d}{2}} C \varepsilon^{\frac{d-2}{2}}\|\hat{\varphi}\|_{H^{1 / 2}(\partial B)} \\
& =\varepsilon^{d-1} C\|\hat{\varphi}\|_{H^{1 / 2}(\partial B)},
\end{aligned}
$$

and the constant $C$ is independent of $\varepsilon$ and $\varphi$.

Note that from the previous lemma and (4.3) it also follows that

$$
\left\|\Upsilon_{\varepsilon} \mathcal{R}_{D_{\varepsilon}} \chi\right\|_{H^{-1 / 2}\left(\partial D_{\varepsilon}\right)} \leq \varepsilon^{\frac{3 d}{2}-1} C\|\hat{\chi}\|_{H^{1 / 2}(\partial B)}
$$

with a constant $C$ that is independent of $\varepsilon$ and $\chi$. Therefore, applying Lemma 5.4 and Lemma 4.2, we can calculate

$$
\begin{aligned}
\left.\frac{\partial w_{\varepsilon}}{\partial \nu}\right|_{\partial D_{\varepsilon}} & =\Upsilon_{\varepsilon}\left(-\frac{1}{2} I+\mathcal{K}_{D_{\varepsilon}}^{N}\right) \chi \\
& =\Upsilon_{\varepsilon}\left(-\frac{1}{2} I+\mathcal{K}_{D_{\varepsilon}}\right) \chi+\Upsilon_{\varepsilon} \mathcal{R}_{D_{\varepsilon}} \chi \\
& =\frac{1}{\varepsilon}\left(\Upsilon\left(\left(-\frac{1}{2} I+\mathcal{K}_{D_{\varepsilon}}\right) \chi\right)^{\wedge}\right)^{\vee}+\mathcal{O}\left(\varepsilon^{\frac{3 d}{2}-1}\right) \\
& =\frac{1}{\varepsilon}\left(\Upsilon\left(-\frac{1}{2} I+\mathcal{K}_{B}\right) \hat{\chi}\right)^{\vee}+\mathcal{O}\left(\varepsilon^{\frac{3 d}{2}-1}\right) .
\end{aligned}
$$

The last term is bounded by $C \varepsilon^{\frac{3 d}{2}-1}\|\hat{\chi}\|_{H^{1 / 2}(\partial B)}$ in $H_{\diamond}^{-1 / 2}\left(\partial D_{\varepsilon}\right)$, where the constant $C$ is independent of $\varepsilon$ and $\chi$. 
Definition 5.6. Define

$$
F: H_{\diamond}^{1 / 2}(\partial B) \rightarrow H_{\diamond}^{-1 / 2}(\partial B), \quad F \varphi:=-\Upsilon\left(-\frac{1}{2} I+\mathcal{K}_{B}\right) \varphi .
$$

Then $F$ is a bounded linear operator, and using Lemma 4.1 we obtain the following asymptotic formula:

Proposition 5.7. For all $\chi \in H_{\diamond}^{1 / 2}\left(\partial D_{\varepsilon}\right)$,

$$
F_{\varepsilon} \chi=\varepsilon^{-1}(F \hat{\chi})^{\vee}+\left(E_{F} \hat{\chi}\right)^{\vee} \text {, }
$$

as $\varepsilon \rightarrow 0$, where the operator $E_{F}$ is bounded by $C \varepsilon^{d-1}$ in the norm of $\mathcal{L}\left(H_{\diamond}^{1 / 2}(\partial B), H_{\diamond}^{-1 / 2}(\partial B)\right)$, and the constant $C$ is independent of $\varepsilon$.

Next we consider the asymptotic behaviour of the operator $L_{\varepsilon}^{*}$ from (2.6). Let $\phi \in H_{\diamond}^{-1 / 2}\left(\partial D_{\varepsilon}\right)$ and $\psi \in H_{\diamond}^{-1 / 2}(\partial \Omega)$. For $X \in\left\{\Omega, B, D_{\varepsilon}\right\}$ we denote by $\langle\cdot, \cdot\rangle_{\partial X}$ the duality pairing between $H_{\diamond}^{1 / 2}(\partial X)$ and $H_{\diamond}^{-1 / 2}(\partial X)$ and use Proposition 5.2 to calculate

$$
\begin{aligned}
\left\langle\phi, L_{\varepsilon}^{*} \psi\right\rangle_{\partial D_{\varepsilon}} & =\left\langle L_{\varepsilon} \phi, \psi\right\rangle_{\partial \Omega} \\
& =\left\langle\varepsilon^{d} L \hat{\phi}+E_{L} \hat{\phi}, \psi\right\rangle_{\partial \Omega} \\
& =\left\langle\hat{\phi}, \varepsilon^{d} L^{*} \psi+E_{L}^{*} \psi\right\rangle_{\partial B} \\
& =\left\langle\phi, \varepsilon\left(L^{*} \psi\right)^{\vee}+\varepsilon^{1-d}\left(E_{L}^{*} \psi\right)^{\vee}\right\rangle_{\partial D_{\varepsilon}},
\end{aligned}
$$

where $L^{*}: H_{\diamond}^{-1 / 2}(\partial \Omega) \rightarrow H_{\diamond}^{1 / 2}(\partial B)$ is the dual operator of $L$.

Recalling Remark 5.3 we obtain the following asymptotic formula:

Proposition 5.8. For all $\psi \in H_{\diamond}^{-1 / 2}(\partial \Omega)$,

$$
L_{\varepsilon}^{*} \psi=\varepsilon\left(L^{*} \psi\right)^{\vee}+\varepsilon^{1-d}\left(E_{L}^{*} \psi\right)^{\vee},
$$

as $\varepsilon \rightarrow 0$, where the operator $E_{L}^{*}$ is bounded by $C \varepsilon^{d+1}$ in the norm of $\mathcal{L}\left(H_{\diamond}^{-1 / 2}(\partial \Omega), H_{\diamond}^{1 / 2}(\partial B)\right)$, and the constant $C$ is independent of $\varepsilon$.

Now we calculate the operator $L^{*}$ explicitly. Let $\phi \in H_{\diamond}^{-1 / 2}(\partial B)$ and $\psi \epsilon$ $H_{\diamond}^{-1 / 2}(\partial \Omega)$. Recalling the definition of the operator $L$ from (5.1) it follows that

$$
\begin{aligned}
\langle L \phi, & \psi\rangle_{\partial \Omega} \\
& =\int_{\partial \Omega} \nabla_{y} N(x, z) \cdot\left(\int_{\partial B} \eta\left(\left(-\frac{1}{2} I+\mathcal{K}_{B}^{*}\right)^{-1} \phi\right)(\eta) \mathrm{d} \sigma(\eta)\right) \psi(x) \mathrm{d} \sigma(x) \\
& =\left(\int_{\partial \Omega} \nabla_{y} N(x, z) \psi(x) \mathrm{d} \sigma(x)\right) \cdot \int_{\partial B} \eta\left(\left(-\frac{1}{2} I+\mathcal{K}_{B}^{*}\right)^{-1} \phi\right)(\eta) \mathrm{d} \sigma(\eta) \\
& =\left(\int_{\partial \Omega} \nabla_{y} N(x, z) \psi(x) \mathrm{d} \sigma(x)\right) \cdot \int_{\partial B} \phi(\xi)\left(\left(-\frac{1}{2} I+\mathcal{K}_{B}\right)^{-1} \eta\right)(\xi) \mathrm{d} \sigma(\xi) .
\end{aligned}
$$

Note that in the last line of this computation $\eta$ is the surface variable on $\partial B$ and therefore $\left(-\frac{1}{2} I+\mathcal{K}_{B}\right)^{-1} \eta$ is defined componentwise for this vector-valued function. Since $N$ is the Neumann function for

$$
\Delta v=0 \quad \text { in } \Omega, \quad \frac{\partial v}{\partial \nu}=\psi \quad \text { on } \partial \Omega
$$


with $\int_{\partial \Omega} v \mathrm{~d} \sigma=0$, we have that

$$
\nabla v(z)=\int_{\partial \Omega} \nabla_{y} N(x, z) \psi(x) \mathrm{d} \sigma(x)
$$

i.e. that

$$
L^{*} \psi=\nabla v(z) \cdot\left(\left(-\frac{1}{2} I+\mathcal{K}_{B}\right)^{-1} \eta\right),
$$

where $v$ is the solution of (5.7).

Now we put our results together and obtain the main result of this paper:

Theorem 5.9. Let $f \in H_{\diamond}^{-1 / 2}(\partial \Omega)$, then

$$
\left(\Lambda_{\varepsilon}-\Lambda_{0}\right) f=\varepsilon^{d} L F L^{*} f+\mathcal{O}\left(\varepsilon^{d+1}\right)
$$

in $H_{\diamond}^{1 / 2}(\partial \Omega)$, as $\varepsilon \rightarrow 0$. More precisely, the last term is bounded by $C \varepsilon^{d+1}\|f\|_{H^{-1 / 2}(\partial \Omega)}$, where the constant $C$ is independent of $\varepsilon$ and $f$.

Proof. From Proposition 5.8 we obtain

$$
L_{\varepsilon}^{*} f=\varepsilon\left(L^{*} f\right)^{\vee}+\varepsilon^{1-d}\left(E_{L}^{*} f\right)^{\vee} .
$$

So by Proposition 5.7 ,

$$
F_{\varepsilon} L_{\varepsilon}^{*} f=\left(F L^{*} f\right)^{\vee}+\varepsilon\left(E_{F} L^{*} f\right)^{\vee}+\varepsilon^{-d}\left(F E_{L}^{*} f\right)^{\vee}+\varepsilon^{1-d}\left(E_{F} E_{L}^{*} f\right)^{\vee} .
$$

With the help of Proposition [5.2, we find for the factorization of $\left(\Lambda_{\varepsilon}-\Lambda_{0}\right) f$ of Lemma 2.1 that

$$
\begin{aligned}
& \left(\Lambda_{\varepsilon}-\Lambda_{0}\right) f=L_{\varepsilon} F_{\varepsilon} L_{\varepsilon}^{*} f=\varepsilon^{d} L F L^{*} f+E_{L} F L^{*} f+\varepsilon^{d+1} L E_{F} L^{*} f \\
& \quad+\varepsilon E_{L} E_{F} L^{*} f+L F E_{L}^{*} f+\varepsilon^{-d} E_{L} F E_{L}^{*} f+\varepsilon L E_{F} E_{L}^{*} f+\varepsilon^{1-d} E_{L} E_{F} E_{L}^{*} f .
\end{aligned}
$$

Now the assertion follows from the estimates in Proposition 5.2 Proposition 5.7 Proposition 5.8, and the continuity of the operators $L, F$, and $L^{*}$.

Figure1 1illustrates the factorization $\Lambda_{\varepsilon}-\Lambda_{0}=L_{\varepsilon} F_{\varepsilon} L_{\varepsilon}^{*}$ from Lemma2.1 and the leading order term $L F L^{*}$ in the corresponding asymptotic factorization $\Lambda_{\varepsilon}-\Lambda_{0}=$ $\varepsilon^{d} L F L^{*}+\mathcal{O}\left(\varepsilon^{d+1}\right)$ from Theorem 5.9.

Finally let $f \in H_{\diamond}^{-1 / 2}(\partial \Omega)$ and let $u_{0}$ be the solution to (2.2). We want to calculate $L F L^{*} f$ explicitly: Since (2.2) and (5.7) coincide, we obtain from (5.8) that

$$
L^{*} f=\nabla u_{0}(z) \cdot\left(\left(-\frac{1}{2} I+\mathcal{K}_{B}\right)^{-1} \eta\right) .
$$

Thus, by applying (5.4),

$$
F L^{*} f=-\nabla u_{0}(z) \cdot \Upsilon \eta=-\nabla u_{0}(z) \cdot \nu(\eta)
$$

where $\nu$ denotes the unit outward normal to $\partial B$, because $\eta_{i}$ is the unique harmonic function on $B$ with Dirichlet data $\left.\eta_{i}\right|_{\partial B}$ for $1 \leq i \leq d$. We deduce from (5.1) that

$$
\begin{aligned}
& L F L^{*} f \\
& \quad=-\nabla_{y} N(\cdot, z) \cdot \int_{\partial B} \eta\left(\left(-\frac{1}{2} I+\mathcal{K}_{B}^{*}\right)^{-1}\left(\nu \cdot \nabla u_{0}(z)\right)\right)(\eta) \mathrm{d} \sigma(\eta) \\
& \quad=-\nabla_{y} N(\cdot, z) \cdot M \nabla u_{0}(z),
\end{aligned}
$$



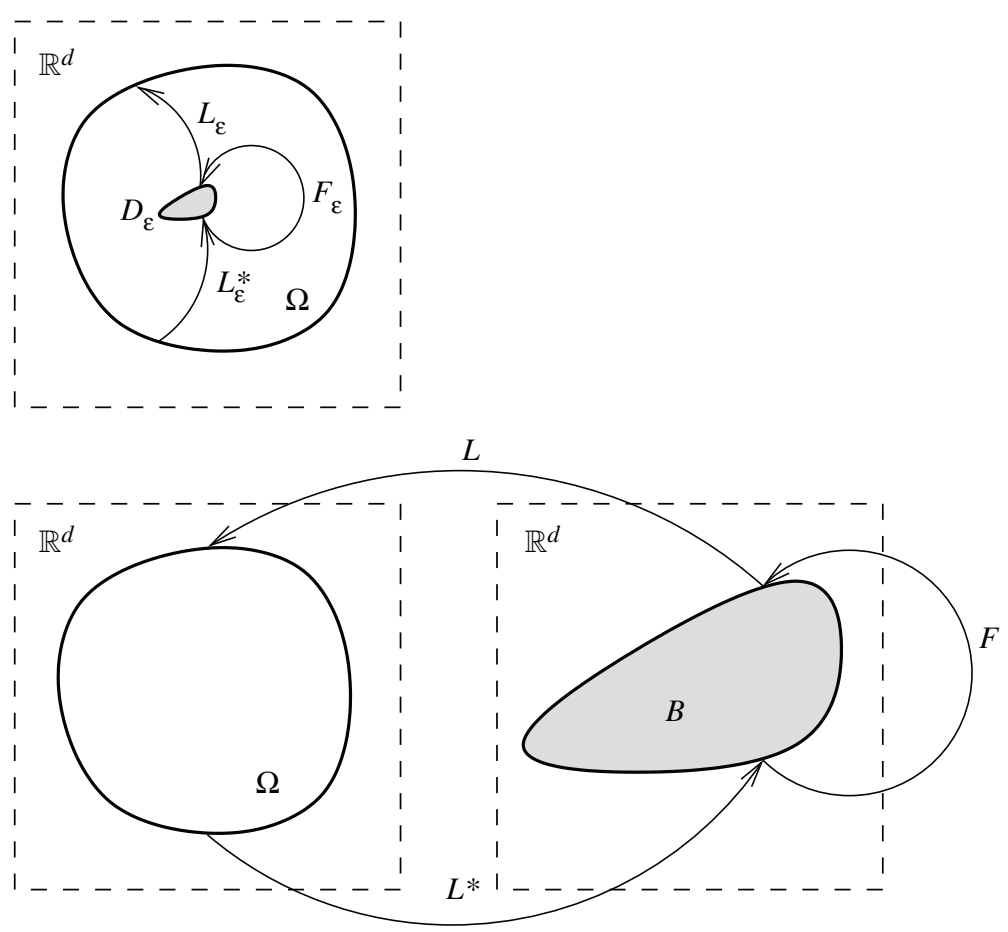

FiguRE 1. Sketch of the factorization $\Lambda_{\varepsilon}-\Lambda_{0}=L_{\varepsilon} F_{\varepsilon} L_{\varepsilon}^{*}$ (above) and of the leading order term $L F L^{*}$ in the corresponding asymptotic factorization $\Lambda_{\varepsilon}-\Lambda_{0}=\varepsilon^{d} L F L^{*}+\mathcal{O}\left(\varepsilon^{d+1}\right)$ (below).

where the matrix $M \in \mathbb{R}^{d \times d}$ is given by $M:=\left(M_{i j}\right)_{i, j=1}^{d}$ with

$$
M_{i j}:=\int_{\partial B} \eta_{i}\left(\left(-\frac{1}{2} I+\mathcal{K}_{B}^{*}\right)^{-1} \nu_{j}\right)(\eta) \mathrm{d} \sigma(\eta)
$$

for $i, j=1, \ldots, d$. $M$ is the so-called polarization tensor of Pólya-Szegö corresponding to the insulating inhomogeneity $D_{\varepsilon}=z+\varepsilon B$. It is a symmetric and negative definite matrix that depends on the relative shape of the inhomogeneity $D_{\varepsilon}$; cf. 6. 24.

We obtain the following corollary:

Corollary 5.10. Let $f \in H_{\diamond}^{-1 / 2}(\partial \Omega)$ and let $u_{0}$ be the solution to (2.2). Then,

$$
\left(\Lambda_{\varepsilon}-\Lambda_{0}\right) f=-\varepsilon^{d} \nabla_{y} N(\cdot, z) \cdot M \nabla u_{0}(z)+\mathcal{O}\left(\varepsilon^{d+1}\right)
$$

in $H_{\diamond}^{1 / 2}(\partial \Omega)$, as $\varepsilon \rightarrow 0$. More precisely, the last term is bounded by $C \varepsilon^{d+1}\|f\|_{H^{-1 / 2}(\partial \Omega)}$, where the constant $C$ is independent of $\varepsilon$ and $f$.

This is exactly the formula derived in 24]; cf. also [5,6].

Remark 5.11. Note that our way of writing the polarisation tensor $M$ differs from that in [24]. But the two expressions are equivalent except that their sign is different, as we will show next: In [24] Friedman and Vogelius define functions $\Psi_{j}$, 
$j=1, \ldots, d$, which are the unique solutions to the exterior problems

$$
\begin{aligned}
\Delta \Psi_{j} & =0, & & \text { in } \mathbb{R}^{d} \backslash \bar{B}, \\
\frac{\partial \Psi_{j}}{\partial \nu} & =-\nu_{j}, & & \text { on } \partial B, \\
\Psi_{j}(\eta) & \rightarrow 0, & & \text { as }|\eta| \rightarrow \infty .
\end{aligned}
$$

Then they define the polarisation tensor $\tilde{M}:=\left(\tilde{M}_{i j}\right)_{i, j=1}^{d}$ by

$$
\tilde{M}_{i j}:=\int_{\partial B} \nu_{i}(\eta)\left(\eta_{j}+\Psi_{j}(\eta)\right) \mathrm{d} \sigma(\eta)
$$

for $i, j=1, \ldots, d$.

Now let $1 \leq i, j \leq d$. If we define $\phi_{j}:=\left(-\frac{1}{2} I+\mathcal{K}_{B}\right)^{-1} \eta_{j}$ and $u_{j}:=\mathcal{D}_{B} \phi_{j}$, we obtain from the jump relation (3.3) that $\left.u_{j}\right|_{\partial B} ^{-}=\eta_{j}$ and $\left.u_{j}\right|_{B}$ is the unique solution to

$$
\Delta u=0 \quad \text { in } B,\left.\quad u\right|_{\partial B} ^{-}=\eta_{j} .
$$

Therefore, $\left.u_{j}\right|_{B}=\eta_{j}$ and we have $\left.\frac{\partial u_{j}}{\partial \nu}\right|_{\partial B} ^{+}=\left.\frac{\partial u_{j}}{\partial \nu}\right|_{\partial B} ^{-}=\nu_{j}$ on $\partial B$. Thus, $-\left.u_{j}\right|_{\mathbb{R}^{d} \backslash \bar{B}}$ solves (5.10), and from the uniqueness of solutions to (5.10) we get $\left.u_{j}\right|_{\mathbb{R}^{d} \backslash \bar{B}}=-\Psi_{j}$. Again from (3.3) we obtain that $\phi_{j}=\left.u_{j}\right|_{\partial B} ^{+}-\left.u_{j}\right|_{\partial B} ^{-}=-\eta_{j}-\left.\Psi_{j}\right|_{\partial B}$. This gives

$$
\begin{aligned}
\tilde{M}_{i j} & =\int_{\partial B} \nu_{i}(\eta)\left(\eta_{j}+\Psi_{j}(\eta)\right) \mathrm{d} \sigma(\eta)=-\int_{\partial B} \nu_{i}(\eta) \phi_{j}(\eta) \mathrm{d} \sigma(\eta) \\
& =-\int_{\partial B} \nu_{i}(\xi)\left(\left(-\frac{1}{2} I+\mathcal{K}_{B}\right)^{-1} \eta_{j}\right)(\xi) \mathrm{d} \sigma(\xi) \\
& =-\int_{\partial B} \eta_{j}\left(\left(-\frac{1}{2} I+\mathcal{K}_{B}^{*}\right)^{-1} \nu_{i}\right)(\eta) \mathrm{d} \sigma(\eta)=-M_{j i}=-M_{i j},
\end{aligned}
$$

where we used the symmetry of $M$. Thus $M=-\tilde{M}$.

\section{Multiple inClusions}

In this section we extend our results to the practically important case of finitely many well separated small inclusions. By that we understand cavities $D_{\varepsilon, i}:=$ $z_{i}+\varepsilon B_{i}$, where $B_{i}$ is a bounded $C^{1, \alpha}$ domain containing the origin, and $1 \leq i \leq m$. The total collection of inclusions thus takes the form $D_{\varepsilon}:=\bigcup_{i=1}^{m}\left(z_{i}+\varepsilon B_{i}\right)$. The points $z_{i} \in \Omega, 1 \leq i \leq m$, that determine the location of the inclusions, are assumed to satisfy

$$
\left|z_{i}-z_{j}\right| \geq c_{0} \quad \forall i \neq j \quad \text { and } \quad \operatorname{dist}\left(z_{i}, \partial \Omega\right) \geq c_{0}
$$

for some constant $c_{0}>0$, and the value of $\varepsilon>0$ is assumed to be sufficiently small. The piecewise constant conductivity distribution is again given by

$$
\sigma_{\varepsilon}(x):= \begin{cases}0, & \text { for } x \in D_{\varepsilon}, \\ 1, & \text { for } x \in \Omega \backslash \overline{D_{\varepsilon}} .\end{cases}
$$

Basically the results and their proofs for a single inclusion from the previous sections can be adopted with few minor modifications which we will comment on now. 
The factorization of the Neumann-to-Dirichlet operator from Lemma 2.1] can be generalized as described in [1]. Therefore, it is convenient to set $\partial D_{\varepsilon}=\partial D_{\varepsilon, 1} \times$ $\cdots \times \partial D_{\varepsilon, m}$ and to interpret the relevant Sobolev spaces accordingly as product spaces, e.g. $H_{\diamond}^{ \pm 1 / 2}\left(\partial D_{\varepsilon}\right)=H_{\diamond}^{ \pm 1 / 2}\left(\partial D_{\varepsilon, 1}\right) \times \cdots \times H_{\diamond}^{ \pm 1 / 2}\left(\partial D_{\varepsilon, m}\right)$. Then the operator $L_{\varepsilon}$ is again defined by (2.3) and (2.4), where the inner Neumann boundary condition should be understood componentwise, i.e. $\frac{\partial v_{\varepsilon}}{\partial \nu}=\phi_{i}$ on $\partial D_{\varepsilon, i}$, for $1 \leq i \leq m$ and $\phi=\left(\phi_{1}, \ldots, \phi_{m}\right) \in H_{\diamond}^{-1 / 2}\left(\partial D_{\varepsilon}\right)$. For the corresponding dual operator $L_{\varepsilon}^{*}$ we again consider the boundary value problem (2.5), whose solution $v_{\varepsilon}^{*}$ is unique up to an additive constant. If we fix an arbitrary solution $v_{\varepsilon}^{*}$ and, for $1 \leq i \leq m$, define $c_{i}:=\int_{\partial D_{\varepsilon, i}} v_{\varepsilon}^{*} \mathrm{~d} \sigma /\left|\partial D_{\varepsilon, i}\right|$, then the dual operator of $L_{\varepsilon}$ is given by

$$
L_{\varepsilon}^{*}: H_{\diamond}^{-1 / 2}(\partial \Omega) \rightarrow H_{\diamond}^{1 / 2}\left(\partial D_{\varepsilon}\right), \quad \psi \mapsto\left(\left.v_{\varepsilon}^{*}\right|_{\partial D_{\varepsilon, 1}}-c_{1}, \ldots,\left.v_{\varepsilon}^{*}\right|_{\partial D_{\varepsilon, m}}-c_{m}\right)
$$

The definition of the operator $F_{\varepsilon}$ remains unchanged if the boundary conditions on $\partial D_{\varepsilon}$ are interpreted componentwise. Then the factorization of $\Lambda_{\varepsilon}-\Lambda_{0}$ stated in Lemma 2.1 holds true in the case of multiple inclusions; cf. 11.

Now we generalize the asymptotic expansions from Section 5 to the case of multiple inclusions. First we again consider the operator $L_{\varepsilon}$ from (2.4). For $\phi=$ $\left(\phi_{1}, \ldots, \phi_{m}\right) \in H_{\diamond}^{-1 / 2}\left(\partial D_{\varepsilon}\right)$ we define $v_{\varepsilon} \in H_{\diamond, \partial \Omega}^{1}\left(\Omega \backslash \overline{D_{\varepsilon}}\right)$ by

$$
v_{\varepsilon}:=\sum_{i=1}^{m} \mathcal{S}_{D_{\varepsilon, i}}^{N} a_{i}
$$

where $a:=\left(a_{1}, \ldots, a_{m}\right) \in H_{\diamond}^{-1 / 2}\left(\partial D_{\varepsilon}\right)$ solves the system of integral equations

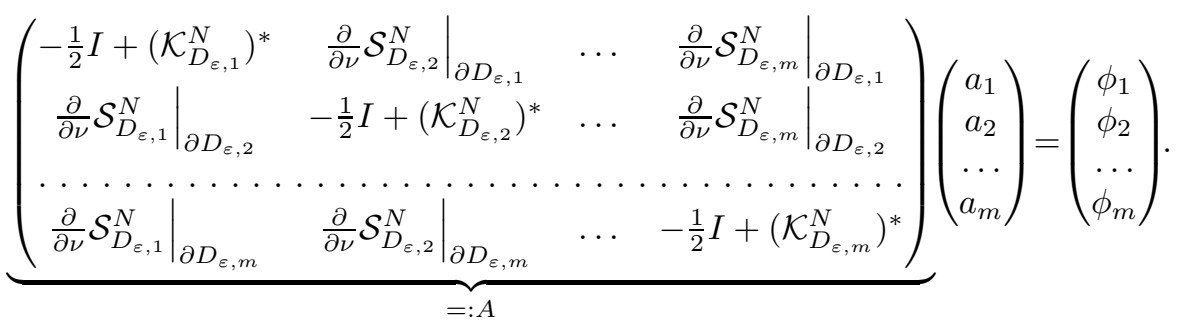

Since the small inclusions are assumed to be well separated from each other and from the boundary $\partial \Omega$, we can estimate the nondiagonal entries of the matrix $A$, using the regularity of $\mathcal{S}_{D_{\varepsilon, i}}^{N} \varphi_{i}$ away from $\partial D_{\varepsilon, i}$, for $1 \leq i \leq m$.

Lemma 6.1. There exists a constant $C$ independent of $\varepsilon$ such that for each $\varphi=$ $\left(\varphi_{1}, \ldots, \varphi_{m}\right) \in H_{\diamond}^{-1 / 2}\left(\partial D_{\varepsilon}\right)$ and $1 \leq i \neq j \leq m$,

$$
\left\|\left.\frac{\partial}{\partial \nu} \mathcal{S}_{D_{\varepsilon, i}}^{N} \varphi_{i}\right|_{\partial D_{\varepsilon, j}}\right\|_{H^{-1 / 2}\left(\partial D_{\varepsilon, j}\right)} \leq \varepsilon^{d-1} C\left\|\varphi_{i}\right\|_{H^{-1 / 2}\left(\partial D_{\varepsilon, i}\right)}
$$


Proof. Let $\varphi=\left(\varphi_{1}, \ldots, \varphi_{m}\right) \in H_{\diamond}^{-1 / 2}\left(\partial D_{\varepsilon}\right)$ and $1 \leq i \neq j \leq m$. Using Lemma 4.1 and the regularity of $\mathcal{S}_{D_{\varepsilon, i}}^{N} \varphi_{i}$ away from $\partial D_{\varepsilon, i}$ we obtain

$$
\begin{aligned}
\left\|\left.\frac{\partial}{\partial \nu} \mathcal{S}_{D_{\varepsilon, i}}^{N} \varphi_{i}\right|_{\partial D_{\varepsilon, j}}\right\|_{H^{-1 / 2}\left(\partial D_{\varepsilon, j}\right)}^{2} \leq \varepsilon^{d} C\left\|\left(\left.\frac{\partial}{\partial \nu} \mathcal{S}_{D_{\varepsilon, i}}^{N} \varphi_{i}\right|_{\partial D_{\varepsilon, j}}\right)^{\wedge j}\right\|_{H^{-1 / 2}\left(\partial B_{j}\right)}^{2} \\
\leq \varepsilon^{d} C\left\|\left(\left.\frac{\partial}{\partial \nu} \mathcal{S}_{D_{\varepsilon, i}}^{N} \varphi_{i}\right|_{\partial D_{\varepsilon, j}}\right)^{\wedge j}\right\|_{L^{2}\left(\partial B_{j}\right)}^{2} \\
=\varepsilon C\left\|\left.\frac{\partial}{\partial \nu} \mathcal{S}_{D_{\varepsilon, i}}^{N} \varphi_{i}\right|_{\partial D_{\varepsilon, j}}\right\|_{L^{2}\left(\partial D_{\varepsilon, j}\right)}^{2} \\
=\varepsilon C \int_{\partial D_{\varepsilon, j}}\left|\int_{\partial D_{\varepsilon, i}} \frac{\partial N(x, y)}{\partial \nu(x)} \varphi_{i}(y) \mathrm{d} \sigma(y)\right|^{2} \mathrm{~d} \sigma(x) \\
\leq \varepsilon C \int_{\partial D_{\varepsilon, j}}\left\|\frac{\partial N(x, \cdot)}{\partial \nu(x)}\right\|_{H^{1 / 2}\left(\partial D_{\varepsilon, i}\right)}^{2}\left\|\varphi_{i}\right\|_{H^{-1 / 2}\left(\partial D_{\varepsilon, i}\right)}^{2} \mathrm{~d} \sigma(x) \\
\leq \varepsilon C \varepsilon^{d-2}\left\|\varphi_{i}\right\|_{H^{-1 / 2}\left(\partial D_{\varepsilon, i}\right)}^{2}\left|\partial D_{\varepsilon, j}\right| \leq \varepsilon^{2 d-2} C\left\|\varphi_{i}\right\|_{H^{-1 / 2}\left(\partial D_{\varepsilon, i}\right)}^{2} .
\end{aligned}
$$

Here $(\cdot)^{\wedge_{j}}$ denotes the usual transformation from (4.1) applied to the $j$-th inhomogeneity $D_{\varepsilon, j}$.

Therefore, $\left.\frac{\partial}{\partial \nu} \mathcal{S}_{D_{\varepsilon, i}}^{N}\right|_{\partial D_{\varepsilon, j}}=\mathcal{O}\left(\varepsilon^{d-1}\right)$ in $\mathcal{L}\left(H_{\diamond}^{-1 / 2}\left(\partial D_{\varepsilon, i}\right), H_{\diamond}^{-1 / 2}\left(\partial D_{\varepsilon, j}\right)\right)$, for $1 \leq$ $i \neq j \leq m$. We deduce that

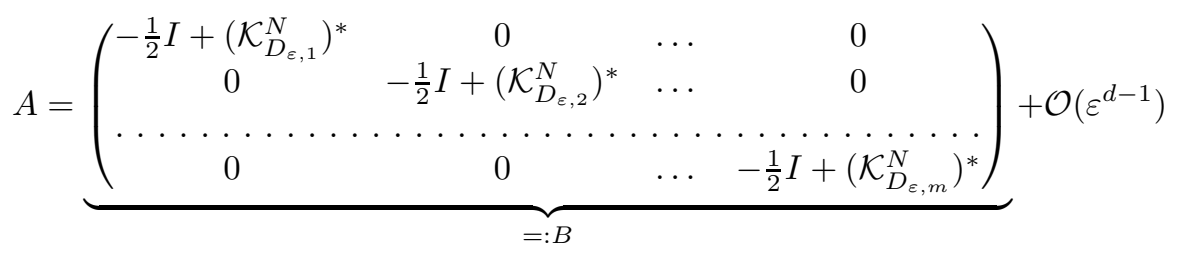

with respect to the maximum row sum of $\mathcal{L}\left(H_{\diamond}^{-1 / 2}\left(\partial D_{\varepsilon, i}\right), H_{\diamond}^{-1 / 2}\left(\partial D_{\varepsilon, j}\right)\right)$-norms, $1 \leq i, j \leq m$. Thus we obtain that $A^{-1}$ exists, and $A^{-1}=B^{-1}+\mathcal{O}\left(\varepsilon^{d-1}\right)$ with respect to the same norm.

Now, calculating along the lines of Section [5 we obtain the following asymptotic formula:

$$
\left.v_{\varepsilon}\right|_{\partial \Omega}=\varepsilon^{d} \sum_{i=1}^{m} \nabla_{y} N\left(\cdot, z_{i}\right) \cdot \int_{\partial B_{i}} \eta\left(\left(-\frac{1}{2} I+\mathcal{K}_{B_{i}}^{*}\right)^{-1} \phi_{i}^{\wedge_{i}}\right)(\eta) \mathrm{d} \sigma(\eta)+\mathcal{O}\left(\varepsilon^{d+1}\right) .
$$

Here again, $(\cdot)^{\wedge^{\wedge}}$ denotes the transformation from (4.1) applied to the $i$-th inclusion $D_{\varepsilon, i}$. The last term is bounded by $C \varepsilon^{d+1} \max _{1 \leq i \leq m}\left\|\phi_{i}^{\wedge^{i}}\right\|_{H^{-1 / 2}\left(\partial B_{i}\right)}$ in $H_{\diamond}^{1 / 2}(\partial \Omega)$, where the constant $C$ is independent of $\varepsilon$ and $\phi$. Therefore, if we define

$$
\begin{aligned}
& L: H_{\diamond}^{-1 / 2}\left(\partial B_{1}\right) \times \cdots \times H_{\diamond}^{-1 / 2}\left(\partial B_{m}\right) \rightarrow H_{\diamond}^{1 / 2}(\partial \Omega), \\
& L \varphi:=\sum_{i=1}^{m} \nabla_{y} N\left(\cdot, z_{i}\right) \cdot \int_{\partial B_{i}} \eta\left(\left(-\frac{1}{2} I+\mathcal{K}_{B_{i}}^{*}\right)^{-1} \varphi_{i}\right)(\eta) \mathrm{d} \sigma(\eta),
\end{aligned}
$$

Proposition 5.2 remains valid in the case of finitely many well separated small inclusions.

Now we return to the diffraction problem (2.7) and the operator $F_{\varepsilon}$ from (2.8). For $\chi=\left(\chi_{1}, \ldots, \chi_{m}\right) \in H_{\diamond}^{1 / 2}\left(\partial D_{\varepsilon}\right)$ we define $w_{\varepsilon}:=\sum_{i=1}^{m} \mathcal{D}_{D_{\varepsilon, i}}^{N} \chi_{i}$. Then, for 
$1 \leq i \leq m$

$$
\left.\frac{\partial w_{\varepsilon}}{\partial \nu}\right|_{\partial D_{\varepsilon, i}}=\Upsilon_{\varepsilon, i}\left(-\frac{1}{2} I+\mathcal{K}_{D_{\varepsilon, i}}^{N}\right) \chi_{i}+\left.\Upsilon_{\varepsilon, i} \sum_{\substack{j=1 \\ j \neq i}}^{m}\left(\mathcal{D}_{D_{\varepsilon, j}}^{N} \chi_{j}\right)\right|_{\partial D_{\varepsilon, i}},
$$

where $\Upsilon_{\varepsilon, i}$ is the interior Dirichlet-to-Neumann operator on $\partial D_{\varepsilon, i}$. As in Lemma 5.5 and Lemma 4.3 we can estimate

$$
\begin{aligned}
\left\|\left(\left.\Upsilon_{\varepsilon, i} \sum_{\substack{j=1 \\
j \neq i}}^{m}\left(\mathcal{D}_{D_{\varepsilon, j}}^{N} \chi_{j}\right)\right|_{\partial D_{\varepsilon, i}}\right)^{\wedge i}\right\|_{H^{-1 / 2}\left(\partial B_{i}\right)} \\
\leq \varepsilon^{-\frac{d}{2}} C \sum_{\substack{j=1 \\
j \neq i}}^{m}\left\|\left.\left(\mathcal{D}_{D_{\varepsilon, j}}^{N} \chi_{j}\right)\right|_{\partial D_{\varepsilon, i}}\right\|_{H^{1 / 2}\left(\partial D_{\varepsilon, i}\right)} \\
\leq \varepsilon^{d-1} C \max _{1 \leq j \leq m}\left\|\chi^{\wedge_{j}}\right\|_{H^{1 / 2}\left(\partial B_{j}\right)},
\end{aligned}
$$

where the constant $C$ is independent of $\varepsilon$ and $\chi$. Therefore, if we define

$$
\begin{aligned}
& F: H_{\diamond}^{1 / 2}\left(\partial B_{1}\right) \times \cdots \times H_{\diamond}^{1 / 2}\left(\partial B_{m}\right) \rightarrow H_{\diamond}^{-1 / 2}\left(\partial B_{1}\right) \times \cdots \times H_{\diamond}^{-1 / 2}\left(\partial B_{m}\right), \\
& F\left(\varphi_{1}, \ldots, \varphi_{m}\right):=\left(-\Upsilon_{1}\left(-\frac{1}{2} I+\mathcal{K}_{B_{1}}\right) \varphi_{1}, \ldots,-\Upsilon_{m}\left(-\frac{1}{2} I+\mathcal{K}_{B_{m}}\right) \varphi_{m}\right),
\end{aligned}
$$

where $\Upsilon_{i}$ is the interior Dirichlet-to-Neumann operator on $\partial B_{i}, 1 \leq i \leq m$, Proposition 5.7 remains valid in the case of finitely many well separated small inclusions.

Calculating along the lines of Section 5 we find that Proposition 5.8 remains valid in the case of finitely many well separated small inclusions, too, and that the adjoint operator

$$
L^{*}: H_{\diamond}^{-1 / 2}(\partial \Omega) \rightarrow H_{\diamond}^{1 / 2}\left(\partial B_{1}\right) \times \cdots \times H_{\diamond}^{1 / 2}\left(\partial B_{m}\right)
$$

of $L$ is given by

$$
L^{*} \psi=\left(\nabla v\left(z_{1}\right) \cdot\left(\left(-\frac{1}{2} I+\mathcal{K}_{B_{1}}\right)^{-1} \eta\right), \ldots, \nabla v\left(z_{m}\right) \cdot\left(\left(-\frac{1}{2} I+\mathcal{K}_{B_{m}}\right)^{-1} \eta\right)\right)
$$

where $v$ is the corresponding solution of (5.7).

Thus we obtain:

Proposition 6.2. Theorem 5.9 holds true in the case of finitely many well separated small inclusions, if $L, F$ and $L^{*}$ are given as in (6.1), (6.2) and (6.3), respectively.

For $1 \leq i \leq m$ let $M^{i}$ denote the polarization tensor corresponding to the $i$-th insulating inclusion $D_{\varepsilon, i}=z_{i}+\varepsilon B_{i}$. In the case of finitely many small inclusions Corollary 5.10 reads as follows:

Corollary 6.3. Let $f \in H_{\diamond}^{-1 / 2}(\partial \Omega)$ and let $u_{0}$ be the solution to (2.2). Then,

$$
\left(\Lambda_{\varepsilon}-\Lambda_{0}\right) f=-\varepsilon^{d} \sum_{i=1}^{m} \nabla_{y} N\left(\cdot, z_{i}\right) \cdot M^{i} \nabla u_{0}\left(z_{i}\right)+\mathcal{O}\left(\varepsilon^{d+1}\right)
$$

in $H_{\diamond}^{1 / 2}(\partial \Omega)$, as $\varepsilon \rightarrow 0$. More precisely, the last term is bounded by $C \varepsilon^{d+1}\|f\|_{H^{-1 / 2}(\partial \Omega)}$, where the constant $C$ is independent of $\varepsilon$ and $f$. 


\section{Determining THE LOCATION OF THE INCLUSIONS}

In this section we again restrict ourselves to the case of a single inhomogeneity $D_{\varepsilon}=z+\varepsilon B$, although we mention that the whole theory also works for multiple inclusions. Moreover, we assume that the boundary $\partial D_{\varepsilon}$ of the inclusion is connected.

The main assertion of the factorization method is the range identity

$$
\mathcal{R}\left(\left(\Lambda_{\varepsilon}-\Lambda_{0}\right)^{1 / 2}\right)=\mathcal{R}\left(L_{\varepsilon}\right),
$$

from which we conclude for the test function

$$
g_{y, d}:=\left.d \cdot \nabla_{y} N(\cdot, y)\right|_{\partial \Omega}, \quad d \in \mathbb{R}^{d},
$$

the following characterization of the inclusion $D_{\varepsilon}$ :

$$
y \in D_{\varepsilon} \quad \text { if and only if } \quad g_{y, d} \in \mathcal{R}\left(\left(\Lambda_{\varepsilon}-\Lambda_{0}\right)^{1 / 2}\right) .
$$

Since $\Lambda_{\varepsilon}-\Lambda_{0}$ is a compact operator (with a range space that is dense in $H_{\diamond}^{1 / 2}(\partial \Omega)$ ), the correct way of implementing (7.2) is via the Picard criterion, i.e. an (infinite) series has to be checked for convergence. We refer to [11,28] for details and numerical implementations.

On the other hand we have shown the asymptotic formula (5.9), i.e. for small values of $\varepsilon$ the operator $\varepsilon^{d} L F L^{*}$ is a good approximation of $\Lambda_{\varepsilon}-\Lambda_{0}$, and hence $\mathcal{R}\left(\Lambda_{\varepsilon}-\Lambda_{0}\right) \approx \mathcal{R}\left(L F L^{*}\right)$. After defining the linear operators

$$
\begin{aligned}
& G: \mathbb{R}^{d} \rightarrow H_{\diamond}^{1 / 2}(\partial \Omega), \quad G a:=\left.a \cdot \nabla_{y} N(\cdot, z)\right|_{\partial \Omega}, \\
& H: H_{\diamond}^{-1 / 2}(\partial B) \rightarrow \mathbb{R}^{d}, \quad H \varphi:=\int_{\partial B} \eta\left(\left(-\frac{1}{2} I+\mathcal{K}_{B}^{*}\right)^{-1} \varphi\right)(\eta) \mathrm{d} \sigma(\eta),
\end{aligned}
$$

an easy computation shows that their dual operators are

$$
\begin{aligned}
& G^{*}: H_{\diamond}^{-1 / 2}(\partial \Omega) \rightarrow \mathbb{R}^{d}, \quad G^{*} \psi=\nabla v(z), \\
& H^{*}: \mathbb{R}^{d} \rightarrow H_{\diamond}^{1 / 2}(\partial B), \quad H^{*} a=a \cdot\left(-\frac{1}{2} I+\mathcal{K}_{B}\right)^{-1} \eta,
\end{aligned}
$$

respectively, where $v$ is the solution of (5.7) and $\eta$ denotes the surface variable on $\partial B$. Then, from (5.1) we find that

$$
L=G H, \quad L^{*}=(G H)^{*}=H^{*} G^{*}, \quad \text { and } \quad L F L^{*}=G H F H^{*} G^{*} .
$$

Recalling the calculations after Theorem 5.9 we see that

$$
H F H^{*}=-M,
$$

where $M$ is the polarization tensor corresponding to the insulating inclusion $D_{\varepsilon}=$ $z+\varepsilon B$, i.e.

$$
L F L^{*}=-G M G^{*}
$$

In [12] it has been proven that

$$
\mathcal{R}\left(L F L^{*}\right)=\mathcal{R}(G)
$$

Therewith one can show that

$$
y=z \quad \text { if and only if } \quad g_{y, d} \in \mathcal{R}\left(L F L^{*}\right) .
$$

Note that $\mathcal{R}(G)$, i.e. $\mathcal{R}\left(L F L^{*}\right)$, is finite dimensional. Hence, instead of using the Picard criterion to check an infinite dimensional range condition we can resort to more familiar techniques from numerical linear algebra and compute, e.g., the angle 
$\theta_{y}$ between $g_{y, d}$ and the range $\mathcal{R}\left(L F L^{*}\right) \approx \mathcal{R}\left(\Lambda_{\varepsilon}-\Lambda_{0}\right)$ in order to implement (7.3) instead of (7.2). We refer to [12,28] for details and numerical implementations.

\section{REFERENCES}

[1] H. Ammari, E. Iakovleva, and D. Lesselier, Two numerical methods for recovering small electromagnetic inclusions from scattering amplitude at a fixed frequency, SIAM J. Sci. Comput. 27 (2005), 130-158. MR2201178 (2006h:35270)

[2] _ A MUSIC algorithm for locating small inclusions buried in a half-space from the scattering amplitude at a fixed frequency, SIAM Multiscale Model. Simul. 3 (2005), 597-628. MR2136165 (2006e:35336)

[3] H. Ammari, E. Iakovleva, D. Lesselier, and G. Perrusson, MUSIC-type electromagnetic imaging of a collection of small three-dimensional bounded inclusions, SIAM J. Sci. Comput., to appear.

[4] H. Ammari, E. Iakovleva, and S. Moskow, Recovery of small inhomogeneities from the scattering amplitude at a fixed frequency, SIAM J. Math. Anal. 34 (2003), 882-900. MR.1969606 (2004a:78022)

[5] H. Ammari and H. Kang, High-order terms in the asymptotic expansions of the steady-state voltage potentials in the presence of conductivity inhomogeneities of small diameter, SIAM J. Math. Anal. 34 (2003), no. 5, 1152-1166. MR2001663 (2004e:35027)

[6] - Reconstruction of Small Inhomogeneities from Boundary Measurements, Lecture Notes in Math., vol. 1846, Springer-Verlag, Berlin, 2004. MR.2168949 (2006k:35295)

[7] B Boundary layer techniques for solving the Helmholtz equation in the presence of small inhomogeneities, J. Math. Anal. Appl. 296 (2004), 190-208. MR2070502 (2005c:35054)

[8] H. Ammari and A. Khelifi, Electromagnetic scattering by small dielectric inhomogeneities, J. Math. Pures Appl. 82 (2003), no. 7, 749-842. MR2005296 (2005a:35262)

[9] H. Ammari, S. Moskow, and M. S. Vogelius, Boundary integral formulae for the reconstruction of electric and electromagnetic inhomogeneities of small volume, ESAIM Control Optim. Calc. Var. 9 (2003), 49-66. MR1957090 (2004j:78025)

[10] H. Ammari, M. S. Vogelius, and D. Volkov, Asymptotic formulas for perturbations in the electromagnetic fields due to the presence of inhomogeneities of small diameter II. The full Maxwell equations, J. Math. Pures Appl. 80 (2001), 769-814. MR1860816 (2003b:78002)

[11] M. Brühl, Explicit characterization of inclusions in electrical impedance tomography, SIAM J. Math. Anal. 32 (2001), 1327-1341. MR.1856252 (2002j:35303)

[12] M. Brühl, M. Hanke, and M. S. Vogelius, A direct impedance tomography algorithm for locating small inhomogeneities, Numer. Math. 93 (2003), 635-654. MR1961882 (2004b:65169)

[13] Y. Capdeboscq and M. S. Vogelius, Optimal asymptotic estimates for the volume of internal inhomogeneities in terms of multiple boundary measurements, Math. Model. Numer. Anal. 37 (2003), no. 2, 227-240. MR1991198(2005c:35067)

[14] A general representation formula for boundary voltage perturbations caused by internal conductivity in homogeneities of low volume fraction, Math. Model. Numer. Anal. 37 (2003), no. 1, 159-173. MR1972656 (2004b:35334)

[15] D. Cedio-Fengya, S. Moskow, and M. S. Vogelius, Identification of conductivity imperfections of small diameter by boundary measurements. Continuous dependence and computational reconstruction, Inverse Problems 14 (1998), no. 3, 553-595. MR1629995 (99d:78011)

[16] M. Cheney, The linear sampling method and the MUSIC algorithm, Inverse Problems $\mathbf{1 7}$ (2001), no. 4, 591-595. MR1861470

[17] D. Colton, J. Coyle, and P. Monk, Recent developments in inverse acoustic scattering theory, SIAM Rev. 42 (2000), no. 3, 369-414. MR.1786932 (2001f:76066)

[18] D. Colton, K. Giebermann, and P. Monk, A regularized sampling method for solving threedimensional inverse scattering problems, SIAM J. Sci. Comput. 21 (2000), no. 6, 2316-2330. MR:1762044 (2000m:65165)

[19] D. Colton, H. Haddar, and P. Monk, The linear sampling method for solving the electromagnetic inverse scattering problem, SIAM J. Sci. Comput. 24 (2002), no. 3, 719-731. MR.1950509 (2003m:78020)

[20] D. Colton and A. Kirsch, A simple method for solving inverse scattering problems in the resonance region, Inverse Problems 12 (1996), no. 4, 383-393. MR1402098(97d:35032) 
[21] M. Costabel, Boundary integral operators on Lipschitz domains: elementary results, SIAM J. Math. Anal. 19 (1988), no. 3, 613-626. MR0937473 (89h:35090)

[22] R. Dautray and J. L. Lions, Mathematical Analysis and Numerical Methods for Science and Technology. Vol. 2. Functional and Variational Methods, Springer-Verlag, Berlin, 1988. MR0969367 (89m:00001)

[23] A. J. Devaney, Super-resolution processing of multi-static data using time reversal and $M U$ $S I C$, preprint.

[24] A. Friedman and M. Vogelius, Identification of small inhomogeneities of extreme conductivity by boundary measurements: a theorem on continuous dependence, Arch. Rational Mech. Anal. 105 (1989), no. 4, 299-326. MR0973245 (90c:35198)

[25] B. Gebauer, The factorization method for real elliptic problems, Z. Anal. Anwend. 25 (2006), 81-102. MR2216883

[26] B. Gebauer, M. Hanke, A. Kirsch, W. Muniz, and C. Schneider, A sampling method for detecting buried objects using electromagnetic scattering, Inverse Problems 21 (2005), 20352050. MR 2183666 (2006k:78009)

[27] V. Girault and P.-A. Raviart, Finite Element Methods for Navier-Stokes Equations. Theory and Algorithms, Springer Series in Computational Mathematics, vol. 5, Springer-Verlag, Berlin, 1986. MR0851383 (88b:65129)

[28] M. Hanke and M. Brühl, Recent progress in electrical impedance tomography, Inverse Problems 19 (2003), S65-S90. MR 2036522

[29] A. Kirsch, Characterization of the shape of a scattering obstacle using the spectral data of the far field operator, Inverse Problems 14 (1998), no. 6, 1489-1512. MR1662460|(99k:35193)

[30] _ The MUSIC algorithm and the factorization method in inverse scattering theory for inhomogeneous media, Inverse Problems 18 (2000), no. 4, 1025-1040. MR1929280 (2003f:35286)

[31] , The factorization method for Maxwell's equations, Inverse Problems 20 (2004), no. 6, S117-S134. MR2107232 (2005i:35285)

[32] _ The factorization method for a class of inverse elliptic problems, Math. Nachr. 278 (2005), no. 3, 258-277. MR2110531 (2006h:35281)

[33] R. Kress, Linear Integral Equations, Applied Mathematical Sciences, vol. 82, Springer-Verlag, Berlin, 1989. MR1007594 (90j:45001)

[34] A factorization method for an inverse Neumann problem for harmonic vector fields, Georgian Math. J. 10 (2003), no. 3, 549-560. MR2023274 (2004m:35276)

[35] W. McLean, Strongly Elliptic Systems and Boundary Integral Equations, Cambridge University Press, Cambridge, 2000. MR1742312 (2001a:35051)

[36] J.-C. Nédélec, Acoustic and Electromagnetic Equations. Integral Representations for Harmonic Problems, Applied Mathematical Sciences, vol. 144, Springer-Verlag, New York, 2001. MR.1822275 (2002c:35003)

[37] M. S. Vogelius and D. Volkov, Asymptotic formulas for perturbations in the electromagnetic fields due to the presence of inhomogeneities of small diameter, Math. Model. Numer. Anal. 34 (2000), 723-748. MR1784483 (2001f:78024)

Centre de Mathématiques Appliquées, CNRS UMr 7641 and Ecole Polytechnique, 91128 Palaiseau Cedex, France

E-mail address: ammari@cmapx.polytechnique.fr

Institut für Mathematik, Johannes Gutenberg-Universität, 55099 Mainz, Germany

E-mail address: griesmaier@math.uni-mainz.de

Institut für Mathematik, Johannes Gutenberg-Universität, 55099 Mainz, Germany

E-mail address: hanke@math.uni-mainz.de 